\title{
Evolución de la comunidad científica de la Dirección Estratégica a partir de la Strategic Management Journal 1980-2009
}

\author{
Guillermo Armando Ronda Pupo - Universidad de Holguín, (Cuba) ${ }^{1}$ \\ Luis Ángel Guerras Martín- Universidad Rey Juan Carlos, (España)
}

\begin{abstract}
Resumen
Se presenta la dinámica de la creación, el desarrollo y la diseminación de la red de la comunidad científica de la disciplina dirección estratégica en torno a la creación y divulgación de conocimientos a través de la revista Strategic Management Journal desde 1980 hasta 2009. Se expone la evolución de la posición de los países participantes en la estructura de la red, los diferentes estadios por los cuales transita la red, la transformación de vértices a nodos y de estos a hubs durante la diseminación de conocimientos de la disciplina dirección estratégica y por último se analiza la influencia del país ubicado en la posición central de la red en la evolución y desarrollo de los países ubicados en la semi-periferia y la periferia a través de la cooperación en la publicación de artículos en la revista mencionada.
\end{abstract}

Palabras clave: Dirección Estratégica - Cienciometría - Redes sociales - Strategic Management Journal, intra-cooperación, inter-cooperación.

\begin{abstract}
The paper presents the dynamics of the creation, development and dissemination of the strategic management scientific community network during knowledge's creation and communication through the Strategic Management Journal from 1980 to 2009. The paper exposes the evolution of the participant countries' position within the network structure. The different stages the network goes through, the vertices' transformation into nodes, and hubs are also presented, and finally, the influence of the country in the core of the network in the development of the countries in the semi-periphery and periphery of the network through the cooperation for publication of articles in SMJ.
\end{abstract}

Key words: Strategic Management - Scientometrics - Social Networks - Strategic Management Journal, intra-cooperation, inter-cooperation.

\footnotetext{
${ }^{1}$ Enviar correspondencia a: Guillermo Armando Ronda Pupo: rondapupo@yahoo.com
} 


\section{Introducción}

La dirección estratégica, como disciplina de investigación académica, comienza a desarrollarse durante la primera mitad de la década de 1960. Las obras pioneras sobre la disciplina fueron: Strategy and Structure: Chapters in the History of American Industrial Enterprise (Chandler, 1962); Corporate Strategy (Ansoff, 1965); Business Policy: Text and Cases (Learned, Christensen, Andrews, y Guth, 1965); The Concept of Corporate Strategy (Andrews, 1971); Strategy Formulation: Analytical Concepts (Hofer y Schendel, 1978). Estas obras se convirtieron en clásicos de la teoría de la dirección estratégica y contribuyeron a introducir y consolidar rápidamente nuevos conceptos dentro del naciente campo de la dirección de empresas.

Aunque en la literatura se pueden encontrar varias definiciones de dirección estratégica, como campo de estudio, se adopta la definición aportada por Nag, Hambrick y Chen. (2007:944) que emerge como una síntesis de su investigación: "el campo de la dirección estratégica trata de las iniciativas intencionadas y emergentes tomadas por los altos directivos en nombre de los propietarios, y que implican la utilización de recursos para mejorar el rendimiento de la empresa en su entorno externo."

Durante el desarrollo de la disciplina se han estudiado varios aspectos específicos de la misma, tales como: la enseñanza de la estrategia en las universidades, principalmente de Estados Unidos (Eldredge y Galloway, 1983; Gordon y Howell, 1959; Summer et al., 1990); la estructura intelectual, principales autores y artículos centrales de la disciplina (Furrer, Thomas, y Goussevskaia, 2008; Nerur, Rasheed y Natarajan, 2008; Ramos-Rodríguez y Ruiz-Navarro, 2004); la definición del constructo y el ámbito de la dirección estratégica como campo de estudio (Feurer y Chaharbaghi, 1995; Fréry, 2006); el estudio e introducción de métodos estadísticos para la investigación de la dirección estratégica (Hahn y Doh, 2006; Hoetker, 2006; Hulland, 1999; Ketchen y Shook, 1996); y la investigación sobre la influencia de las revistas relevantes en el desarrollo de la disciplina ( Tahai y Meyer, 1999; Phelan, Ferreira, y Salvador, 2002; Podsakoff, MacKenzie, Bachrach, y Podsakoff, 2005; Azar y Brock, 2008).

Todas las tendencias mencionadas contribuyeron a la generación de conocimientos y a un rápido y sostenido crecimiento de la producción científica de la disciplina, favoreciendo así la creación y consolidación de la estructura intelectual y la generación de un cuerpo teórico sólido para convertir la dirección estratégica en una disciplina científica dentro de las ciencias sociales. 
En este resultado ha tenido un papel protagonista la revista Strategic Management Journal (en adelante SMJ) que, con su aparición en 1980, marcó un hito en la historia de la evolución de la comunidad científica que investiga en la disciplina. Esta revista se convirtió en la líder en la creación y diseminación del conocimiento en este campo específico de investigación científica y en una de las primeras revistas sobre dirección de empresas en general. En el año 2010 SMJ cumple 30 años de publicación ininterrumpida y creciente de artículos especializados sobre dirección estratégica. Este período de tiempo es suficiente para permitir la identificación y visualización de la red de países en torno a la disciplina a partir del estudio de su producción científica.

En la literatura revisada aparece una brecha que dificulta responder los siguientes interrogantes: ¿Cómo se ha conformado y evolucionado la estructura de la red de la comunidad científica de la dirección estratégica en torno a la revista SMJ?, ¿cuáles son las etapas y sus características por las cuales ha transitado la estructura de la red durante la construcción y diseminación del conocimiento sobre dirección estratégica?, ¿cuáles son las características de la red de investigadores en dirección estratégica en torno a la revista SMJ?, ¿cómo ha influido la cooperación entre autores en la incorporación de nuevos países, en la creación de conocimiento sobre dirección estratégica, su desarrollo y posterior diseminación?, ¿qué nivel de significación tiene la cooperación entre los países ubicados en las áreas de semiperiferia y/o periferia con los países del núcleo de la red en este proceso?

La respuesta a estas preguntas agregará información, para complementar la ya existente, sobre la estructura intelectual de la dirección estratégica como campo de investigación al aportar la visualización del mapa de la conformación, crecimiento y transformación de la estructura de la red de la comunidad científica en torno a la revista SMJ a los 30 años de haberse creado.

En la literatura revisada no aparecen evidencias relacionadas con el nacimiento, evolución y diseminación de la comunidad científica del campo de la dirección estratégica. Por tanto, este trabajo complementa a otros trabajos sobre esta disciplina como campo científico de investigación, lo que constituye su principal aportación tratando de cubrir así el desfase identificado.

Los objetivos del artículo son: (1) establecer la dinámica de la evolución de la comunidad científica de la dirección estratégica durante la creación, desarrollo y diseminación de conocimientos a través de la revista SMJ; (2) establecer las diferentes etapas y características por las que atraviesa la red de la comunidad científica de la dirección estratégica a lo largo de su evolución; (3) determinar el 
nivel de significación de la cooperación inter-países para la generación, desarrollo y diseminación de conocimientos en la disciplina.

\section{Revisión de literatura}

El artículo se basa en algunos trabajos recientes sobre la evolución de la disciplina dirección estratégica, que se pueden segmentar en dos grupos diferentes: un grupo trata de delinear la evolución de la estructura intelectual de la dirección estratégica mientras que el otro ha centrado su atención en la influencia de revistas en el desarrollo de la disciplina. Trataremos de exponer las contribuciones más significativas de las investigaciones de ambos grupos y las brechas que aún permanecen en la literatura.

En el primer grupo varios estudios han analizado la evolución y desarrollo de la dirección estratégica como disciplina científica utilizando diversas técnicas cuantitativas. Así, se ha estudiado la definición de la propia disciplina (Nag et al., 2007), su estructura intelectual tanto a través de los artículos más relevantes (Ramos-Rodríguez y Ruiz-Navarro, 2004; Furrer et al., 2008) como a través de los autores más influyentes (Nerur et al., 2008; Furrer et al., 2008) y las líneas y temas de investigación predominantes (Furrer et al., 2008).

Especialmente interesantes son los tres artículos relacionados con la estructura intelectual de la disciplina y que se asemejan en: (1) la unidad de análisis utilizada, (2) los métodos de investigación empleados, (3) el marco temporal escogido. Por ejemplo, Ramos-Rodríguez y Ruiz-Navarro (2004) estudiaron 21 años de producción científica de SMJ (1980-2000) segmentados en 3 etapas de 7 años cada una. Para ello emplearon la técnica bibliométrica de co-citaciones apoyada con el método estadístico Escalamiento Multidimensional. Sus resultados principales fueron determinar los cambios en la estructura intelectual de la disciplina y los autores más relevantes de cada etapa.

Como conclusiones importantes de este trabajo se pueden señalar las siguientes: a) importancia muy destacada de los autores clásicos en la primera etapa, tanto los procedentes de la teoría de la organización como de los pioneros en la enseñanza de la estrategia en las escuelas de negocios; b) pérdida progresiva de influencia de Ios autores clásicos; c) relevancia de la economía industrial a partir de la segunda etapa, especialmente a partir de los trabajos de Porter (1980, 1985); y d) aparición y desarrollo del enfoque basado en los recursos que se constituye en la contribución más importante a la disciplina en la tercera etapa (Ramos-Rodríguez y RuizNavarro, 2004). 
Como complemento al trabajo anterior, Nerur et al. (2008) estudian 21 años de producción científica publicada en SMJ (1980-2000) segmentados en 3 etapas. Furrer et al. (2008) estudian 26 años de producción científica publicada en las revistas Academy of Management Journal, Academy of Management Review, Administrative Science Quarterly y SMJ (1980-2005) segmentados en 5 etapas.

Nag et al. (2007) estudian un marco temporal de 21 años de artículos sobre dirección estratégica en las revistas Academy of Management Journal, Academy of Management Review y Administrative Science Quarterly (1980-2000), segmentados en 5 etapas. Todos ellos toman como año inicial de referencia 1980, lo cual viene determinado, en gran medida, por la aparición en dicho año de la revista de referencia en la disciplina, es decir, SMJ.

Todos los trabajos anteriormente señalados han empleado como unidad de análisis el artículo o sus palabras clave para estudiar la estructura intelectual de la dirección estratégica y su evolución, así como sus autores y contribuciones más relevantes. Sin embargo, no hemos encontrado ningún trabajo relacionado con la evolución de la comunidad científica de la disciplina. Por ello parece necesario analizar esta evolución a través de las diferentes etapas de su desarrollo histórico.

El presente trabajo se asemeja a los anteriores en el marco temporal estudiado y la inclusión de SMJ como una de las fuentes básicas para el estudio de la disciplina. No obstante, se diferencia en: (1) la unidad de análisis empleada (países), y (2) los métodos empleados (análisis de redes sociales combinados con la técnica bibliométrica del análisis de co-palabras).

En el segundo grupo se encuentran los artículos que han centrado su atención en la influencia de las revistas en el desarrollo de la dirección estratégica. En este grupo se encuentran importantes contribuciones como los trabajos de Franke et al. (1990), Tahai y Meyer (1999), Phelan et al. (2002), Podsakoff et al. (2005).

Franke et al. (1990) muestran la evolución de la dirección estratégica y la relación entre la calidad de las revistas y el impacto de los artículos relacionados con este campo de estudio. Asimismo, muestran la correlación entre la medición de la calidad, el factor de impacto de los artículos y la influencia de la revista. Tahai y Meyer (1999) estudian 17 revistas sobre dirección de empresas, tomando para ello las citas recibidas por las mismas en 1993 y 1994 . El resultado obtenido fue la introducción y el empleo de una nueva metodología que posibilitó ubicar en un ranking las revistas estudiadas. El artículo confirma el papel protagonista de SMJ, no sólo entre las revistas de dirección estratégica, sino entre las de la dirección de empresas en general. 
Phelan et al. (2002) analizan el papel de SMJ mostrando su evolución durante sus primeros 20 años de existencia y demuestran su protagonismo como espacio de conformación, desarrollo y diseminación del conocimiento en dirección estratégica. Azar y Brock (2008) publicaron un ránking de la revistas sobre dirección estratégica basado en índices de citaciones. Dicho ránking sitúa a SMJ en el primer lugar.

El estudio de Podsakoff et al. (2005) aporta las revistas más influyentes en el campo de la dirección estratégica. Los autores estudian las citaciones de 28 revistas durante un espacio temporal de 20 años. El resultado mostró que las revistas con mayor índice de crecimiento fueron la Academy of Management Journal, la Academy of Management Review y SMJ. Este resultado sitúa a SMJ entre las primeras revistas en el mundo en el campo de la dirección de empresas.

Todos estos estudios se asemejan en la unidad de análisis que utilizan (las revistas) y se diferencian en los métodos empleados. Las principales diferencias de nuestro estudio en relación con este segundo grupo de trabajos radican en la unidad de análisis y los métodos empleados.

En el campo de la cienciometría, Katz (1994) y Wagner y Leydesdorff (2005a,b) presentaron resultados sobre estudios relacionados con la estructura de la red de la ciencia en la colaboración internacional. Wagner y Leydesdorff $(2005 a, b)$ elaboran mapas de la colaboración empleando datos de la co-autoría en el nivel internacional de todos los campos de la ciencia en los años 1990 y 2000. Katz (1994) presenta, específicamente, un estudio sobre la relación entre la distancia geográfica y el incremento de la cooperación en el nivel intra-nacional. Para ello emplea datos de la co-autoría de artículos por autores de universidades de Canadá, Australia y Reino Unido. En el presente estudio, aunque se emplea la co-autoría en ambos niveles (intra-nacional e internacional) para recopilar la información, se emplean técnicas del análisis de redes sociales para visualizar la evolución de la estructura de una comunidad científica perteneciente a un subcampo de la dirección que no había sido estudiada con anterioridad.

Este estudio pretende determinar los patrones de la dinámica de la comunidad científica de la disciplina dirección estratégica durante la creación, desarrollo y diseminación del conocimiento relacionado con esta disciplina en torno a la SMJ, descubrir los centros de difusión y los patrones en las relaciones entre países durante la incorporación de los mismos a la estructura de la red, así como determinar el nivel de significación de la cooperación inter-países en su evolución. 


\section{Método}

\section{Unidad de análisis}

Para este estudio se utiliza como unidad de análisis cada uno de los países representados en los artículos publicados en SMJ entre 1980 y 2009, ambos inclusive. A estos efectos, se ha considerado el país al que pertenece la institución vinculada con cada uno de los autores de cada artículo. Es importante señalar, por tanto, que no se ha tenido en cuenta la nacionalidad de cada autor, dado que esa información no está disponible, sino la de la institución a la que representan.

\section{Muestra}

La muestra la constituyen todos los artículos publicados en SMJ desde el volumen 1 número 1 de 1980 hasta el volumen 30, número 13 del año 2009. Se excluyeron del estudio las notas editoriales y la revisión de libros. Las razones de la elección de SMJ como fuente para el estudio son las siguientes: (1) se ubica como la primera revista de la disciplina dirección estratégica y entre las 3 primeras revistas en el campo de la dirección de empresas en el mundo de acuerdo con el Journal of Citation Reports (JCR); (2) todos los artículos se relacionan directamente con la disciplina dirección estratégica; (3) SMJ ha mantenido un crecimiento sostenido de su factor de impacto durante los últimos cuatro años según el Journal of Citation Reports, Social Sciences Citation Index.

\section{Marco temporal}

El marco temporal del estudio abarca desde 1980 hasta 2009 (30 años), ambos inclusive. Si bien la comunidad científica de la dirección estratégica comienza a formarse a partir de 1960, el crecimiento de la producción científica es significativo a partir de 1980 con la aparición de la revista de referencia de la disciplina, SMJ. Se selecciona este rango temporal teniendo en cuenta que la fuente del estudio es SMJ que surge en el año 1980. Este rango de tiempo se estratificó en tres etapas de diez años cada una: la primera comprende los años 1980 a 1989, la segunda los años 1990 a 1999, y la tercera los años 2000 a 2009. Este rango de 10 años se considera adecuado ya que representa un segmento significativo de tiempo que es testigo de las fases de crecimiento y madurez en una comunidad académica.

\section{Procesamiento}

El procesamiento consta de 3 pasos: (1) la recogida de información, (2) la creación de la red para cada etapa estudiada y (3) el análisis de la red a través de técnicas de análisis de redes sociales combinado con técnicas bibliométricas de análisis de co-palabras. 
Paso 1. Recogida y codificación de la información: Para recopilar la información se revisaron todos los artículos publicados durante el marco temporal del estudio. Se realizó el recuento de la cantidad de autores procedentes de cada país por artículo durante los diez años que conforman cada etapa. Para codificar la información de cada año se cuantificó la cantidad de autores por cada artículo y se asignó 1 punto al país de procedencia por cada autor que participa en el mismo. Cuando participan autores de dos o más países se le asigna un punto por cada autor a cada país representado. En la cuantificación de la información de cada país se respetó el país declarado explícitamente por el autor del artículo.

Se emplea este método de recuento para evitar los problemas que se presentan al cuantificar la colaboración entre instituciones o países mediante el método de fraccionamiento $1 / n_{\text {th }}$ descritos en Katz (1994). Como los datos procedentes del Social Science Citation Index no aportan información individual sobre la afiliación institucional para cada autor por separado se realizó este recuento directamente revisando cada uno de los artículos.

El proceso de recopilación de la información sobre la cooperación entre autores y países se realizó en dos pasos. Primero, se codificó cada uno de los artículos publicados durante el período del estudio y se le asignó un número que representa el volumen, el número y la posición del artículo en el número. Así el 010101 correspondería al primer artículo del número uno del volumen 1 de la SMJ. Además se identifica cada artículo con el nombre de su primer autor, lo que evita la posible duplicación de información que conduciría a errores en el procesamiento. Con la información recopilada se creó una matriz de publicación (artículos $\times$ países).

Muchos autores estandarizan a continuación la matriz creando una matriz de coocurrencia (Katz, 1994) o la simetrizan empleando el cálculo de coeficientes como Cosine, Jaccar, Pearson, Inclusión, Proximidad u otros (Wagner y Leydesdorff, 2005a), como una forma de reducir las diferencias ocasionadas por el dominio de países muy grandes. Sin embargo, en el presente estudio se han empleado las frecuencias obtenidas para cada país para crear directamente las redes de cada etapa utilizando el software Pajek. Para ello se sitúa el valor de la cantidad de autores por cada país como valor vectorial del mismo y la cantidad de artículos publicados en co-autoría como dato para calcular el valor de las líneas que conectan a los países unidos por dicha cooperación. A continuación se realiza el cálculo del grado de centralidad de cada país. 


\section{Paso 2. Creación de la red para cada etapa}

En el campo de la dirección de empresas, el análisis de redes sociales ha sido empleado y desarrollado por Burt $(2001,2007,2008)$. Igualmente, ha sido utilizado en trabajos relacionados con las alianzas estratégicas (Gulati, 1998; Gulati et al, 2000; Zajac, 1998; Kogut, 2000). Para crear la red para cada etapa y su posterior estudio se realizaron los siguientes pasos:

1) Se creó una random network con el uso del software Pajek con los países que aparecen representados en los artículos publicados en SMJ en cada una de las etapas estudiadas. Para ello se empleó el comando Net>Random network>Total No. of Arcs.

2) Se realizó una partición para colocar el nombre de los países a cada vértice que conforma la estructura de la red para cada etapa. Para ello se empleó el comando Partition $>$ Create a ramdom partition $>1$-Mode. De esta manera ya se introducen los datos en una matriz simétrica o de modo 1.

3) Se creó un vector constante para ubicar los datos de los valores correspondientes a la cantidad de autores de cada país que publicaron artículos en cada etapa estudiada. Para ello se utilizó el comando Vector>Create a Constant Vector.

4) Se asignaron los valores de las líneas que conectan a cada par de países que son representados mediante la co-autoría de artículos publicados en la SMJ. En la red, cada valor de las líneas se corresponde con la cantidad de artículos publicados mediante la cooperación (internacional). Para asignar el valor final de cada línea se emplea el método de colaboración bilateral descrito por Katz (1994). Por ejemplo un artículo que se publica con la participación de autores de 4 países $A, B, C$ y $D$ se contaría como la asistencia de 6 colaboraciones bilaterales, A-B, A-C, A-D, B-C, B-D y C-D. La fórmula para el cálculo del valor de las líneas sería $c=[n(n / 1)] / 2(K a t z$, 1994:35).

Para representar el valor correspondiente a la cooperación entre autores procedentes de instituciones de un mismo país (intra-cooperación) se emplean loops. El valor asignado a cada loop se corresponde con la cantidad de artículos publicados mediante intra-cooperación.

Paso 3. Análisis de la información: con la información procedente del paso anterior se realiza el análisis de la red para cada etapa. El análisis consta de 4 fases.

3.1 Ubicación de los países en relación con su grado de centralidad en zonas de la red. En ocasiones, para visualizar la estructura de una red con el fin 
de explorar los patrones de la actividad de colaboración mediante los vínculos de co-autoría, se utiliza el escalamiento multidimensional, lo cual tiende a propiciar resultados distorsionados (Peters y Van Raan, 1993a,b; Katz, 1994). Además de para evitar esta dificultad, hemos empleado técnicas del análisis de redes sociales por dos razones principales: 1) permiten determinar los índices de densidad, conectividad, clusterabilidad y centralización, que son útiles para trazar la evolución de la red a lo largo de los periodos analizados, 2) al poder crear las redes directamente en Pajek no es necesario simetrizar la matriz, lo cual favorece la utilización de los datos reales para cada país. Al realizar cualquier correlación o cálculos de similitud para simetrizar matrices de modo dos, los datos reales para cada vértice se pierden ya que los resultados de cualquiera de estos cálculos se convierten en el valor de las líneas y/o relaciones.

Una vez que se crean las redes para cada una de las etapas analizadas, se determina el grado de centralidad de cada país que participa en la estructura de la red para cada etapa. Con esta información se analiza la estabilidad y la evolución de la centralidad de cada país participante en la estructura de la red durante cada etapa. La medida de centralidad es un conjunto de algoritmos que se calcula en cada red y que permite determinar tanto su estructura como la posición de cada vértice en la red. (De Nooy, Mrvar y Batagelj, 2005).

Para analizar posteriormente los resultados obtenidos, se llevan a cabo dos tipos de acciones. Primero, se ubica a cada país en las áreas de la estructura de la red (núcleo, semi-periferia o periferia). Para este paso se emplean los valores resultantes del cálculo del grado de centralidad de cada país. Dado que estos valores varían en un rango entre 0,00 y 1,00, dicho rango se estratificó en tres segmentos o estratos. En el primero se ubican los países que pertenecen a la periferia de la red, con un grado de centralidad entre 0,00 y 0,33. En el segundo se ubican los países pertenecientes a la semi-periferia de la red, con un grado de centralidad entre 0,34 y 0,67 . Finalmente, el tercer estrato recoge los países que pertenecen al núcleo de la red y que tienen un grado de centralidad entre 0,68 y 1,00 .

Una vez ubicado cada país, de acuerdo con su grado de centralidad, en la zona que le corresponde, se traza su evolución en relación con los cambios de posición que sufre durante las 3 etapas estudiadas. Los resultados del análisis se visualizan mediante el empleo del software Pajek (Batagelj y Mrvar, 1996). Para ello se emplea el comando vector del software Pajek y se realiza la partición por intervalos 
seleccionando "selected thresholds". Finalmente se energiza la red mediante la técnica Kamada y Kawai (1989).

\subsection{Determinación del estadio de desarrollo de cada país en la estructura de}

la red. Para determinar la evolución de cada país durante cada etapa se calcularon los índices de emisión y de recepción. El índice de emisión se calculó como el cociente entre el número de autores del país emisor que publicaron artículos como co-autores y el total de autores de dicho país que publicaron artículos en SMJ en la etapa analizada. El índice de recepción se calculó como el cociente entre el número de autores del país receptor que publicaron artículos como co-autores y el total de autores de dicho país que publicaron artículos en SMJ en la etapa analizada.

A partir del cálculo de estos índices, de la ubicación en los diferentes estratos o posiciones en la estructura de la red y de las características y patrones observados durante las tres etapas, se definieron los estadios de desarrollo por los que puede atravesar cada país: (1) incorporación/conformación; consolidación/diseminación y (3) expansión/ transformación (tabla 1).

Tabla 1 Etapas de evolución de la red de la comunidad científica de la dirección estratégica

\begin{tabular}{ll}
\hline \multicolumn{1}{c}{ Etapa } & \multicolumn{1}{c}{ Características } \\
\hline Conformación & - No aparecen los tres estratos de una red. \\
/Incorporación & - Aparecen muchos vértices aislados. \\
& - La dirección de la relación es del núcleo hacia la periferia. \\
& - Bajos niveles de densidad y conectividad de la red. \\
& - Predominio del índice de recepción sobre el de emisión. \\
\hline & - Incorporación de nuevos vértices a la estructura de la red. \\
& - Disminución de la cantidad de vértices aislados. \\
& - Se inicia el desarrollo de los vértices ubicados en la periferia. \\
& - Algunos vértices pasan de la periferia a la semi-periferia. \\
Consolidación/ & - Se incrementa la cantidad de vínculos entre vértices ubicados \\
diseminación & en la zona del núcleo con otros ubicados en zona de la periferia. \\
& - Se inicia la aparición de vínculos entre vértices ubicados en la \\
& semi-periferia con vértices ubicados en la periferia. \\
& - Crece la densidad y conectividad de la red. \\
& - Se transforman vértices en nodos. \\
& - Predominio del índice de recepción sobre el de emisión. \\
& - Aparecen los 3 estratos de una red. \\
& - Crecimiento estable de vértices de la red. \\
& - Se transforman nodos en hubs diseminadores y obtienen \\
& mayor índice de emisión que de recepción. \\
& - Se definen los vértices de la semi-periferia y la periferia. \\
Expansión/ & - Crece la relación entre vértices ubicados en la semi-periferia. \\
transformación & - Aparecen relaciones entre vértices ubicados en la zona de la \\
& periferia. \\
& - La estructura de la red tiende a ser completa. \\
& - Surgen sub-redes adyacentes a los hubs y crece la estructura \\
de la red.
\end{tabular}


3.3 Evolución de las redes para cada etapa. Para estudiar la evolución de la red de la comunidad científica en torno a la revista SMJ para cada etapa se dieron dos pasos. El primero consistió en determinar los índices de densidad y centralidad de la red en cada etapa y en el segundo se determinó el índice de disimilitud.

La densidad indica el número de líneas en una red y refleja la coherencia interna o fuerza de las relaciones entre los integrantes de la misma. Se calcula dividiendo el total de relaciones presentes entre el total de relaciones posibles. El índice de conectividad evidencia la cantidad de conexiones existentes en la red en relación con la cantidad de relaciones posibles. Una red completa ocurre cuando todos los vértices que conforman la misma están conectados entre sí, es decir, no existen vértices aislados.

La comparación de las redes obtenidas para las tres etapas estudiadas se realizó a través del método de tres pasos propuesto por Callon Courtial y Laville, (1991) adaptado a redes integradas por países. La comparación de dos redes en tiempos diferentes se realiza mediante el cálculo del índice de disimilitud (Callon et al.,

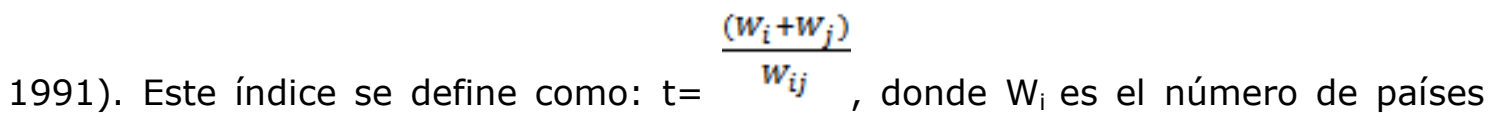
presentes en el clúster $C_{i} ; W_{j}$ es el número de países presentes en el clúster $C_{j} ; y$ $\mathrm{W}_{\mathrm{ij}}$ es el número de países comunes a $\mathrm{C}_{\mathrm{i} \text { y }} \mathrm{C}_{\mathrm{j}}$.

La transformación de las redes y su intersección con otras redes a través de periodos de tiempos proporciona evidencias de la emergencia de comunidades de investigación. La similitud de las redes en diferentes etapas se calcula a través del índice de similitud (Coultier, Monarch, y Konda, 1998).

Además, para poner de manifiesto la evolución de cada país en la estructura de la red, se tienen en cuenta tres niveles: vértices, nodos y hubs Wagner y Leydesdorff (2005b). El vértice es la unidad más pequeña en la estructura de la red. Un nodo es un vértice al cual se conectan otros vértices y un hub es un nodo muy bien conectado a otros nodos y vértices y que, por lo general, se visualiza como una subred dentro de la estructura de la red. Se entiende por transformación el momento en que un vértice dentro de la red transita de un estadio a otro superior; por ejemplo, de vértice a nodo o de nodo a hub.

\subsection{Determinación de la influencia de la cooperación en el desarrollo de la} comunidad científica en torno a SMJ: Wagner y Leydesdorff, (2005b) consideran que la colaboración internacional en la ciencia es una red de comunicación diferente a la de un sistema nacional y que posee una dinámica interna propia. 
Para la presente investigación se tuvo en cuenta la definición de colaboración universidad-universidad brindada por Katz (1994). Así, la cooperación se entiende como la participación conjunta de autores procedentes de dos o más instituciones de un mismo país (intra-nacional) o de instituciones de dos o más países (internacional) compartiendo esfuerzos y beneficios para alcanzar un objetivo común, en este caso reflejado en la publicación de artículos en la SMJ. En este paso se realizó la comparación de los resultados de los países que mostraron crecimiento sostenido y que a su vez crecieron en su cooperación con el país ubicado en el núcleo de la red con países que crecieron pero con poca cooperación con el país ubicado en esta zona para determinar el nivel de significación de la transformación ocurrida en los mismos. Para ello se utilizó la prueba estadística t.

\section{Análisis y discusión de los resultados}

Entre 1980 y 1987, en la revista SMJ, (volúmenes 1 al 8) aparecieron 4 números por cada volumen. En esta etapa se inicia la incorporación de números especiales dedicados a temas específicos de desarrollo dentro de la disciplina. En 1988 la cantidad de números por volumen crece de 4 a 6 y en 1990 a 7. Entre 1991 y 1995 publicó 8 números por volumen y en 1999 se estabiliza la aparición de 12 números ordinarios y dos números especiales por cada volumen.

El crecimiento paulatino de la cantidad de números por volumen favoreció el incremento de la cantidad de artículos publicados y con ello la posibilidad de incorporación de autores de nuevos países, por lo que se va conformando una red de la comunidad científica cada vez más amplia y compleja. Esto muestra que SMJ se convirtió en un espacio especializado para la generación, desarrollo y diseminación del conocimiento sobre dirección estratégica y a la vez un nexo para crear y desarrollar una red de cooperación entre investigadores dentro de la comunidad científica de la disciplina en el mundo.

La calidad de las publicaciones de SMJ fue incrementando su valor de manera sostenida, lo cual queda evidenciado a través del crecimiento de su factor de impacto (ver figura 1 ) y de su posición en el ranking de revistas sobre dirección de empresas en general y en el de las de dirección estratégica en particular. 


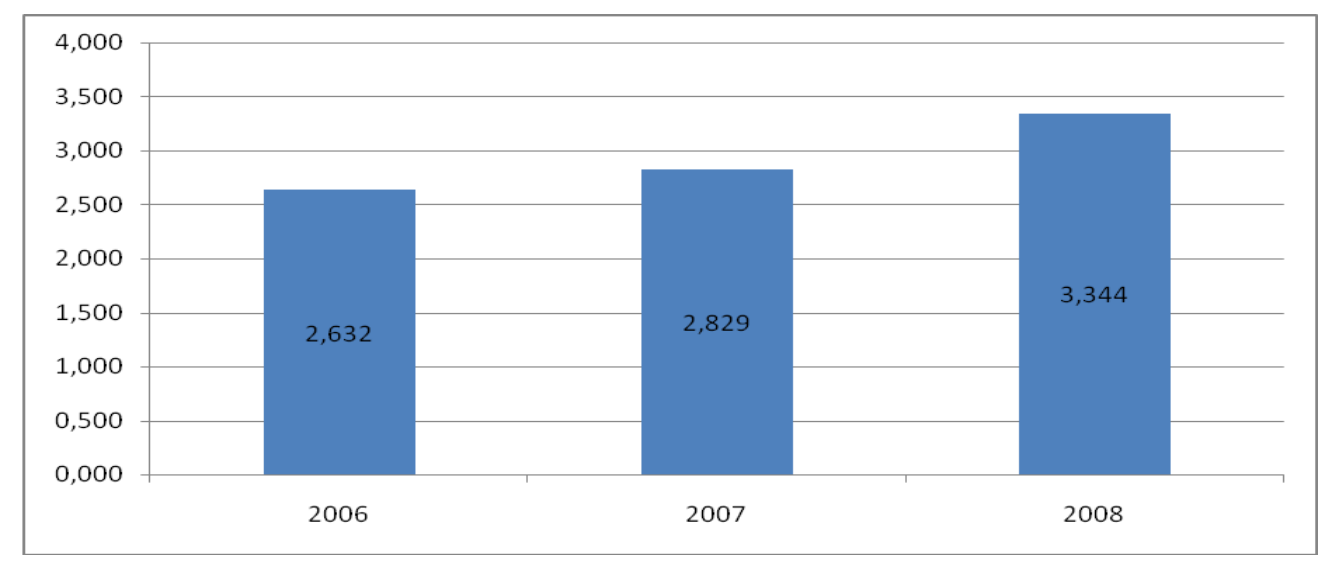

Figura 1. Evolución del factor de impacto de SMJ.

Fuente: Journal Citation Report- Social Sciences Citation Index.

Entre 1980 y 2009 publicaron artículos en SMJ 3.145 autores de 38 países. De estos autores, el $72.9 \%$ son de Estados Unidos, el 5.88\% de Reino Unido, el $4.36 \%$ de Canadá y el $3.59 \%$ de Francia, $1.37 \%$ de Hong Kong, $1.30 \%$ de España y $1.08 \%$ de Holanda. La suma de la participación de los autores del resto de los 34 países representados en la red es menor de un $1 \%$ por cada uno.

De los 1.616 artículos publicados en el marco temporal estudiado, el $32.24 \%$ fue creado por un solo autor. Los datos describen un decrecimiento sostenido de la cantidad de artículos publicados en esta categoría. En la etapa I (1980 a 1989) el $49,08 \%$ del total de los artículos fueron publicados por un solo autor. En la etapa II (1990 a 1999), el 33.05\% y en la etapa III (2000 a 2009) el porcentaje fue del $23.76 \%$. Este dato evidencia un crecimiento del número de artículos publicados en SMJ mediante la co-autoría.

Tabla 2. Estadísticas descriptivas para cada etapa.

\begin{tabular}{lcccccccc}
\hline \multirow{2}{*}{ Países } & \multicolumn{2}{c}{ Etapa I } & \multicolumn{2}{c}{ Etapa II } & \multicolumn{2}{c}{ Etapa III } & \multicolumn{2}{c}{ TOTAL } \\
\cline { 2 - 10 } & Total & $\%$ & Total & $\%$ & Total & $\%$ & Total & $\%$ \\
\hline Alemania & 1 & $0,19 \%$ & 7 & $0,62 \%$ & 15 & $1,00 \%$ & 23 & $0,73 \%$ \\
\hline Argentina & 0 & $0,00 \%$ & 0 & $0,00 \%$ & 1 & $0,07 \%$ & 1 & $0,03 \%$ \\
\hline Australia & 0 & $0,00 \%$ & 3 & $0,27 \%$ & 13 & $0,87 \%$ & 16 & $0,51 \%$ \\
\hline Austria & 0 & $0,00 \%$ & 0 & $0,00 \%$ & 3 & $0,20 \%$ & 3 & $0,10 \%$ \\
\hline Bélgica & 3 & $0,57 \%$ & 5 & $0,45 \%$ & 7 & $0,47 \%$ & 15 & $0,48 \%$ \\
\hline Canadil & 0 & $0,00 \%$ & 0 & $0,00 \%$ & 2 & $0,13 \%$ & 2 & $0,06 \%$ \\
\hline Corea & 36 & $6,86 \%$ & 35 & $3,12 \%$ & 66 & $4,41 \%$ & 137 & $4,36 \%$ \\
\hline Chile & 0 & $0,00 \%$ & 3 & $0,27 \%$ & 21 & $1, .40 \%$ & 24 & $0,76 \%$ \\
\hline China & 0 & $0,00 \%$ & 2 & $0,18 \%$ & 0 & $0,00 \%$ & 2 & $0,06 \%$ \\
\hline Chipre & 0 & $0,00 \%$ & 1 & $0,09 \%$ & 10 & $0,67 \%$ & 11 & $0,35 \%$ \\
\hline Dinamarca & 0 & $0,00 \%$ & 0 & $0,00 \%$ & 3 & $0,20 \%$ & 3 & $0,10 \%$ \\
\hline Emiratos Árabes Unidos & 0 & $0,00 \%$ & 0 & $0,00 \%$ & 4 & $0,27 \%$ & 4 & $0,13 \%$ \\
\hline
\end{tabular}




\begin{tabular}{|c|c|c|c|c|c|c|c|c|}
\hline Estados Unidos & 391 & $74,48 \%$ & 875 & $77,99 \%$ & 1.027 & $68,56 \%$ & 2.293 & $72,91 \%$ \\
\hline España & 3 & $0,57 \%$ & 4 & $0,36 \%$ & 34 & $2,27 \%$ & 41 & $1,30 \%$ \\
\hline Finlandia & 1 & $0,19 \%$ & 0 & $0,00 \%$ & 14 & $0,93 \%$ & 15 & $0,48 \%$ \\
\hline Francia & 19 & $3,62 \%$ & 43 & $3,83 \%$ & 51 & $3,40 \%$ & 113 & $3,59 \%$ \\
\hline Grecia & 0 & $0,00 \%$ & 1 & $0,09 \%$ & 3 & $0,20 \%$ & 4 & $0,13 \%$ \\
\hline Hong Kong & 0 & $0,00 \%$ & 6 & $0,53 \%$ & 37 & $2,47 \%$ & 43 & $1,37 \%$ \\
\hline India & 0 & $0,00 \%$ & 1 & $0,09 \%$ & 1 & $0,07 \%$ & 2 & $0,06 \%$ \\
\hline Indonesia & 0 & $0,00 \%$ & 1 & $0,09 \%$ & 0 & $0,00 \%$ & 1 & $0,03 \%$ \\
\hline Irlanda & 2 & $0,38 \%$ & 1 & $0,09 \%$ & 0 & $0,00 \%$ & 3 & $0,10 \%$ \\
\hline Israel & 6 & $1,14 \%$ & 11 & $0,98 \%$ & 5 & $0,33 \%$ & 22 & $0,70 \%$ \\
\hline Italia & 0 & $0,00 \%$ & 2 & $0,18 \%$ & 8 & $0,53 \%$ & 10 & $0,32 \%$ \\
\hline Japón & 1 & $0,19 \%$ & 6 & $0,53 \%$ & 14 & $0,93 \%$ & 21 & $0,67 \%$ \\
\hline Macau & 0 & $0,00 \%$ & 0 & $0,00 \%$ & 2 & $0,13 \%$ & 2 & $0,06 \%$ \\
\hline Nueva Zelanda & 1 & $0,19 \%$ & 5 & $0,45 \%$ & 3 & $0,20 \%$ & 9 & $0,29 \%$ \\
\hline Noruega & 0 & $0,00 \%$ & 8 & $0,71 \%$ & 6 & $0,40 \%$ & 14 & $0,45 \%$ \\
\hline Portugal & 1 & $0,19 \%$ & 0 & $000 \%$ & 4 & $0,27 \%$ & 5 & $0,16 \%$ \\
\hline Rusia & 0 & $0,00 \%$ & 0 & $0,00 \%$ & 1 & $0,07 \%$ & 1 & $0,03 \%$ \\
\hline Singapur & 0 & $0,00 \%$ & 2 & $0,18 \%$ & 22 & $1,47 \%$ & 24 & $0,76 \%$ \\
\hline Sudáfrica & 1 & $0,19 \%$ & 0 & $0,00 \%$ & 0 & $0,00 \%$ & 1 & $0,03 \%$ \\
\hline Suecia & 1 & $0,19 \%$ & 10 & $0,89 \%$ & 16 & $1,07 \%$ & 27 & $0,86 \%$ \\
\hline Suiza & 2 & $0,38 \%$ & 4 & $0,36 \%$ & 9 & $0,60 \%$ & 15 & $0,48 \%$ \\
\hline Taiwán & 0 & $0,00 \%$ & 1 & $0,09 \%$ & 3 & $0,20 \%$ & 4 & $0,13 \%$ \\
\hline Holanda & 6 & $1,14 \%$ & 17 & $1,52 \%$ & 11 & $0,73 \%$ & 34 & $1,08 \%$ \\
\hline Turquía & 3 & $0,57 \%$ & 0 & $0,00 \%$ & 1 & $0,07 \%$ & 4 & $0,13 \%$ \\
\hline Reino Unido & 47 & $8,95 \%$ & 68 & $6,06 \%$ & 70 & $4,67 \%$ & 185 & $5,88 \%$ \\
\hline Total & 525 & $100,00 \%$ & 1,122 & $100,00 \%$ & 1.498 & $100,00 \%$ & 3.145 & $100,00 \%$ \\
\hline
\end{tabular}

En la etapa I participaron 18 países liderados claramente por los autores de instituciones de los Estados Unidos. En la segunda etapa, se incorporaron 12 nuevos países a la red mientras que 4 que estuvieron en la primera etapa ya no aparecen. Este resultado evidencia un crecimiento neto de un $44.44 \%$ y la participación de 26 países en la comunidad científica de esta disciplina. En la tercera etapa la red crece a 34 países, lo que representa un crecimiento del $30.77 \%$ respecto de la etapa anterior. Mientras que 8 nuevos países se incorporaron, 3 se reincorporaron y 3 se retiraron de la red (tabla 2). La figura 2 muestra la evolución de la red con un crecimiento estable de la cantidad de países incorporados a la comunidad científica de dirección estratégica. 


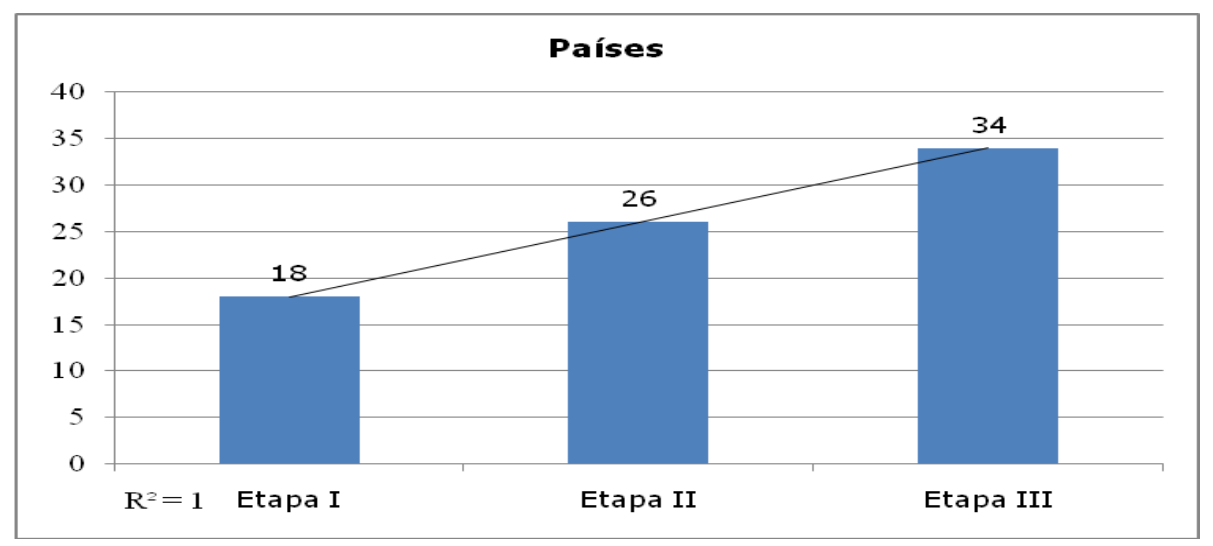

Figura 2. Evolución de la incorporación de países a la red de la comunidad científica de la dirección estratégica.

También crece de manera estable durante las tres etapas analizadas la cooperación de autores inter-países. La relación de cooperación fluyó inicialmente de autores del país central hacia países ubicados en la zona de la periferia de la red. Esta tendencia favoreció el crecimiento de la red con la incorporación de autores de nuevos países, lo que propició además el inicio de la propagación de los conocimientos generados a todos los países participantes en la red.

\section{Caracterización de la etapa I: 1980-1989}

Durante la primera etapa, 525 autores de 18 países publicaron trabajos en SMJ. De ellos, el $74,48 \%$ fueron autores procedentes de universidades norteamericanas. Esta tendencia obedece, esencialmente, a la madurez de la comunidad científica, en torno a la dirección estratégica, de ese país que desarrollaba la introducción de conceptos y modelos desde la década de 1960. Este protagonismo situó la comunidad científica norteamericana de dirección estratégica en la zona del núcleo en la estructura de la red durante la segunda etapa de su evolución.

La densidad de la red, en esta etapa es de 0,05 y la conectividad de 5,86. El $50,00 \%$ de los países incorporados aparecen aislados. Esta tendencia muestra que inicialmente la cooperación fluye entre autores de instituciones de un mismo país favoreciendo el desarrollo endógeno de la comunidad científica de cada país, elemento propio de la fase de conformación de una escuela de pensamiento en un área de investigación.

Durante la etapa I, 22 artículos fueron publicados en cooperación. De ellos, 16 con la participación de autores de universidades de Estados Unidos $(72,72 \%), 4$ con la participación de autores de universidades de Reino Unido, 1 de Canadá y 1 de Francia. Este resultado muestra claramente el predominio de la dirección de las relaciones de cooperación desde Estados Unidos semi-periferia hacia los países ubicados en la periferia (ver figura 3). De acuerdo con los valores de centralidad 
alcanzados por los países en la estructura de la red se visualizan dos áreas: semiperiferia (Estados Unidos) y la periferia (el resto de los países).

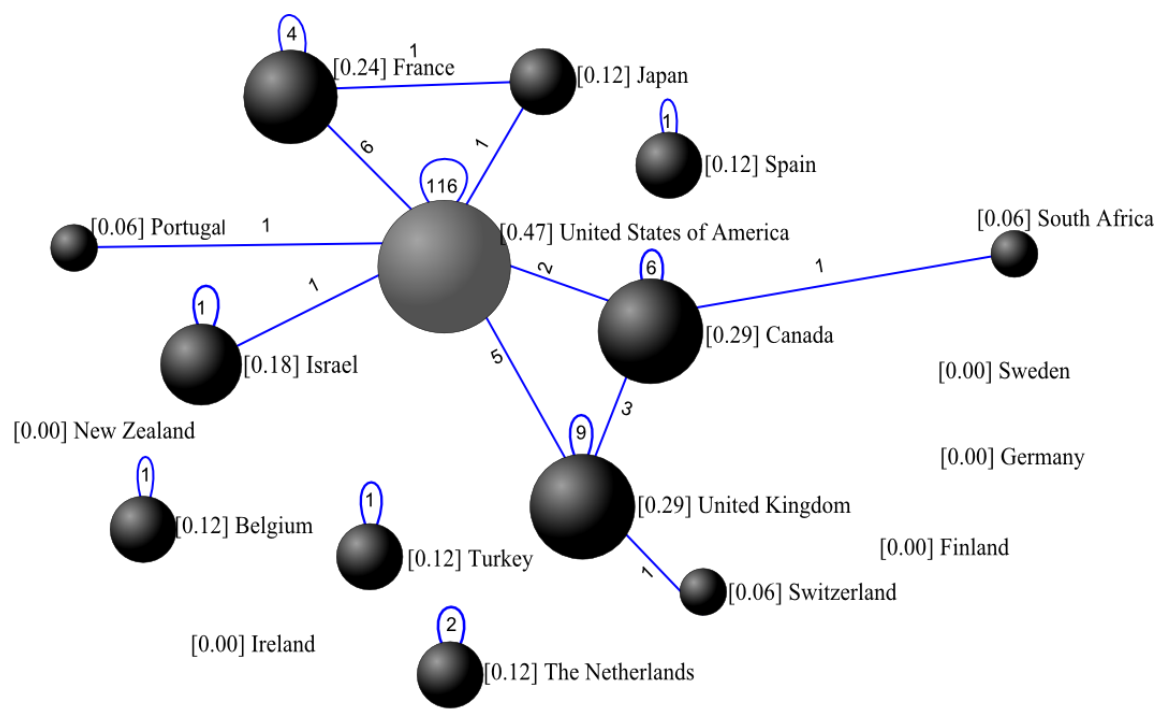

Figura 3. Estructura de la red de países participantes en la Etapa I.

Nota: los valores de los vértices significan el grado de centralidad. El color cambia en relación con la zona en que se ubica cada vértice en la red. Gris oscuro =semi periferia; negro $=$ periferia. Los valores de los loops se corresponden con la cantidad de artículos en coautoría por intra-cooperación.

\section{Caracterización de la etapa II: 1990-1999}

En la etapa II participaron 1.122 autores de 26 países, lo que representa un crecimiento de un $88,85 \%$ respecto de la etapa anterior. De éstos, el $77,89 \%$ proceden de instituciones norteamericanas. Esto permite que este país evolucione de la semi-periferia y pase a conformar el núcleo de la red. El crecimiento del número total de autores en esta etapa obedece, entre otras posibles causas, al crecimiento de la cantidad de números por volumen de la revista SMJ. Además, si bien 4 países desaparecen de la red, la incorporación de 12 nuevos países permite observar que la estructura general de la red experimenta crecimiento apreciable.

El nivel de densidad crece de 0,05 a 0,12 y el de conectividad de 3,81 a 8,35. Este crecimiento obedece a que la cantidad de países aislados decrece de 9 a 3 y la cantidad de artículos publicados en co-autoría crece. Además, los patrones de las relaciones fluyen desde el país ubicado en el núcleo hacia la periferia y comienza a diseminarse la cooperación con una pequeña participación de la relación entre autores de países ubicados en la periferia (figura 4).

En la etapa II 77 artículos fueron publicados en cooperación internacional, lo cual muestra un crecimiento de un $71,5 \%$ respecto de la etapa anterior. El 78,94\% de los artículos fue publicado con la cooperación de autores de Estados Unidos, por lo 
que se mantiene un nivel similar a la etapa anterior. Crecen de 5 a 15 los artículos publicados mediante la cooperación de países ubicados en la periferia. Se desataca Reino Unido y se mantienen estables Canadá y Francia, por lo que consolidan su posición en la periferia de la red.

Se incrementa la diseminación de la producción científica hacia otros países. Se mantiene el patrón predominante de la dirección de la relación desde el núcleo de la red hacia la periferia y se inicia la relación entre los países que están en la periferia. Este resultado evidencia que la estructura de la red en esta etapa conforma, al igual que en la etapa anterior, dos zonas. A diferencia de la primera, en esta ocasión Estados Unidos evoluciona al núcleo por lo que en esta etapa las áreas de la estructura de la red son núcleo y periferia. También se puede observar el inicio del tránsito de la red del estadio de conformación al de consolidación.

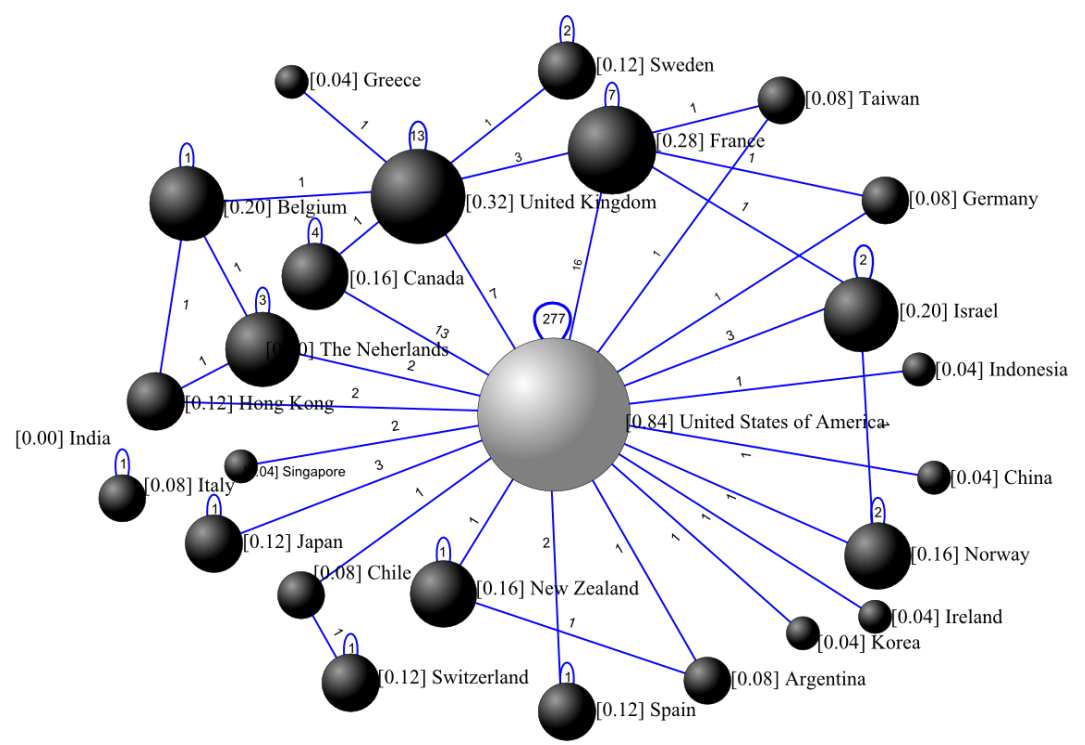

Figura 4. Estructura de la red de países participantes en la etapa II.

El color cambia en relación con la zona en que se ubica cada vértice en la red. Gris claro = núcleo, negro = periferia. Los valores de los loops se corresponden con la cantidad de artículos en coautoría por intra-cooperación.

\section{Caracterización de la etapa III: 2000-2009}

En esta etapa participaron 1.498 autores de 34 países. Al igual que en las etapas anteriores, predominan los autores procedentes de instituciones de Estados Unidos, el $68,56 \%$ del total. En esta etapa se incorporan autores de 11 nuevos países y 3 países que participaron en la anterior ya no aparecen. Esto muestra una presencia algo inestable de los países ubicados en la periferia de la red ya que sólo el 34,21\% (13 países) de la red mantuvo una presencia estable durante las tres etapas. El resto aparece o desaparece en alguna de las etapas. 
Se observa un incremento de la densidad de la red de 0,12 a 0,14 al igual que la conectividad que crece de 9,70 a 13,83. 3 países aparecen aislados, lo cual representa el $8,82 \%$ de la red y significa un decrecimiento de un $2,71 \%$ respecto de la etapa precedente.

En la etapa III, 167 artículos fueron publicados en colaboración internacional, lo cual muestra un crecimiento de un $54.51 \%$ respecto de la etapa 2 y de un $629,09 \%$ respecto a la primera etapa. El $73,61 \%$ de estos artículos fueron publicados con la participación de autores de instituciones de Estados Unidos; el 11,81\% con autores de instituciones de Reino Unido y el 4,86\% de autores de instituciones de Canadá. Este resultado muestra la estabilidad de la posición en el núcleo de la red, aunque disminuye el porcentaje de cooperación de autores de instituciones del país en relación con las etapas anteriores.

En esta etapa, de acuerdo con el grado de centralidad alcanzado por los países, se estratifica la estructura de la red en tres áreas o zonas, núcleo, semi-periferia y periferia (ver figura 5). En la nueva zona de semi-periferia se consolida Reino Unido convirtiéndose en hub y Canadá se incorpora a esta zona e inicia su evolución a nodo diseminador. Francia también se incorpora en esta zona y también gana seguidores procedentes de la periferia de la red.

Como se aprecia, existió un crecimiento de la cantidad de países incorporados a la red durante las 3 etapas, lo cual favoreció la incorporación de nuevos autores y el crecimiento de la cantidad de autores de los países previamente incorporados en la red. Además, esto contribuyó a la diseminación de conocimientos sobre la dirección estratégica y la conformación de esta disciplina en el mundo.

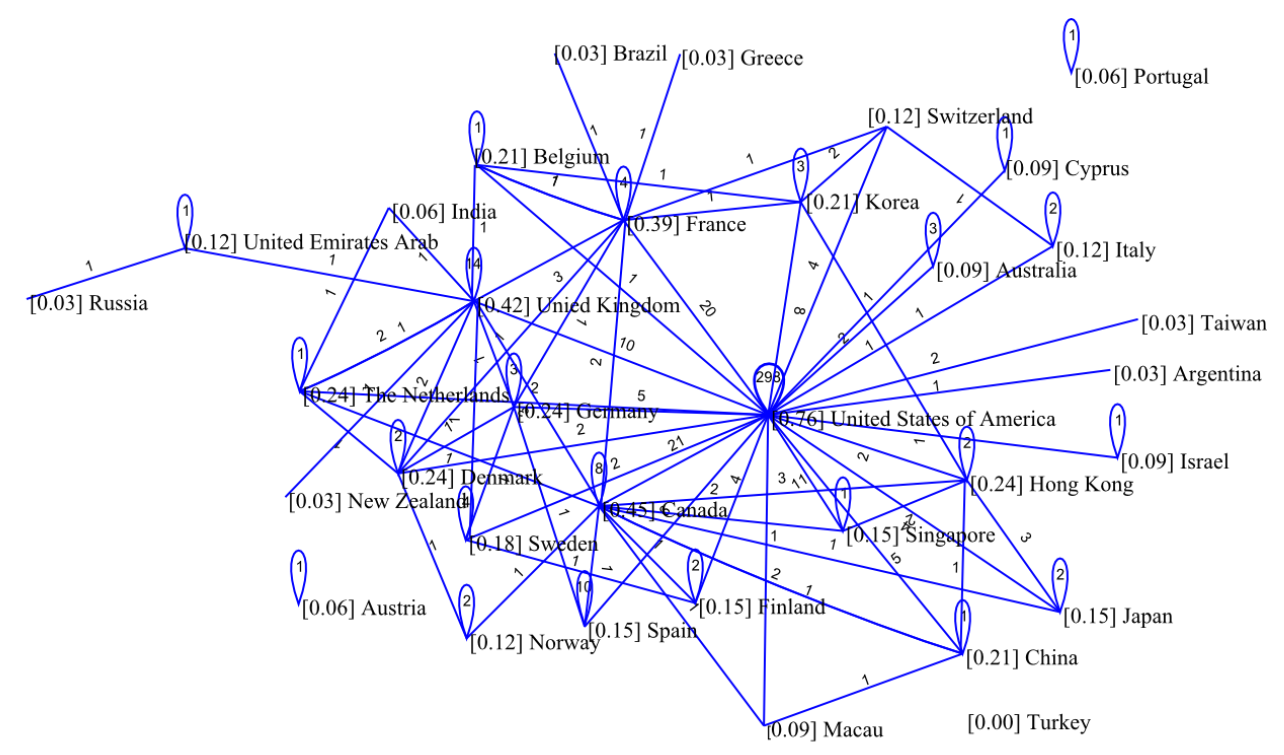

Figura 5. Imagen de la red de países participantes en la Etapa III. 
Los resultados obtenidos muestran que la dinámica de la evolución de la red en torno a la generación y diseminación de conocimiento sobre dirección estratégica posee dos patrones esenciales: (1) la conformación y evolución de la posición que ocupa cada vértice, nodo o hub en la estructura de la red y (2) la etapa de evolución por la que transita la red.

En cuanto a la posición de cada vértice, nodo o hub, el resultado mostró que en un primer momento sólo aparecen claramente la zona semi-periferia, dominada por la comunidad científica de Estados Unidos, y con menor fuerza la zona de periferia con una posición de ventaja de las comunidades científicas de Reino Unido, Canadá y Francia.

En un segundo momento de la evolución de la estructura de la red, y como resultado del avance del grado de centralidad de la comunidad científica de Estados Unidos, esta evoluciona y conforma el núcleo de la red. Sin embargo, el avance de la comunidad científica de los países más avanzados de la periferia (Canadá, Francia y Reino Unido), en este segundo momento, no alcanzan aún el grado de centralidad necesario para avanzar y ubicarse en la semi-periferia.

Durante el tercer momento evolutivo de la red, a partir del crecimiento del grado de centralidad de los países de la semi-periferia, la estructura se estratifica en tres zonas, el núcleo, dominado por Estados Unidos, surge la semi-periferia a través de la evolución de Reino Unido, Canadá y Francia y el resto de los países se ubican en la zona de periferia. Se observa también que algunos países ubicados en la zona de periferia crecen de manera sostenida y se aproximan a la zona de la semi-periferia: Alemania, España, Holanda y Hong Kong.

La evolución de la estructura de la red y los países que la conforman durante las tres etapas analizadas permitió elucidar que transitaron por 3 estadios: (1) conformación/incorporación; (2) consolidación/diseminación; y (3) expansión/ transformación.

Para medir la evolución de cada país en la estructura de la red durante las etapas estudiadas primero se calculó el grado de centralidad de cada país y luego los índices de emisión y de recepción. El resultado de estos índices permitió determinar la evolución de cada país en la estructura de la red, así como establecer la trasformación de vértice a nodo y de nodo a $h u b$.

\section{Grado de centralidad de los países en la estructura de la red}

En la tabla 3 se presenta el grado de centralidad para cada país durante su tránsito en la estructura de la red en las etapas estudiadas. Los 13 países que participaron 
en la red de la comunidad científica de la dirección estratégica durante todo el espacio temporal estudiado describen 3 patrones:

1. Países que su grado de centralidad creció de manera estable y sostenida durante las 3 etapas: Alemania, Bélgica, España, Francia, Holanda, Japón, Reino Unido, Suecia y Suiza.

2. Países que su grado de centralidad creció en la segunda etapa y volvió a decrecer en la tercera: Israel, Nueva Zelanda y Estados Unidos.

3. Países que en la segunda etapa su grado de centralidad decreció, y volvió a crecer en la tercera: Canadá.

El incremento significativo del grado de centralidad de Canadá, Francia y Reino Unido favoreció que en la tercera etapa la estructura de la red se estratificara en tres áreas; núcleo, semi-periferia y periferia. También evidencia el tránsito por los tres niveles de un vértice dentro de la red hasta alcanzar su eslabón más alto. Es decir, estos países se convierten en hubs al crearse una sub red adyacente a los mismos e inician la diseminación de conocimientos favoreciendo, de esta manera, el crecimiento de la red de la comunidad científica en torno a la SMJ por el mundo.

Tabla 3. Grado de centralidad de los países por etapas.

\begin{tabular}{lccc}
\hline & \multirow{2}{*}{ Países } & \multicolumn{3}{c}{ Grado de centralidad } \\
\cline { 2 - 4 } Alemania & Etapa I & Etapa II & Etapa III \\
\hline Argentina & 0,00 & 0,08 & 0,24 \\
\hline Australia & - & - & 0,03 \\
\hline Austria & - & - & 0,09 \\
\hline Bélgica & - & - & 0,06 \\
\hline Brasil & 0,12 & 0,20 & 0,21 \\
\hline Canadá & - & - & 0,03 \\
\hline Chile & 0,29 & 0,16 & 0,45 \\
\hline China & - & 0,08 & - \\
\hline Chipre & - & 0,04 & 0,21 \\
\hline Corea & - & - & 0,09 \\
\hline Dinamarca & - & 0,04 & 0,21 \\
\hline Emiratos Árabes Unidos & - & - & 0,24 \\
\hline España & - & - & 0,12 \\
\hline Estados Unidos de América & 0,12 & 0,12 & 0,15 \\
\hline Finlandia & 0,47 & 0,84 & 0,76 \\
\hline Francia & 0,00 & - & 0,15 \\
\hline Grecia & 0,24 & 0,28 & 0,39 \\
\hline Holanda & - & 0,04 & 0,03 \\
\hline Hong Kong & 0,12 & 0,20 & 0,24 \\
\hline India & - & 0,12 & 0,24 \\
\hline & - & 0,00 & 0,06 \\
\hline
\end{tabular}




\begin{tabular}{lccc} 
Indonesia & - & 0,04 & - \\
\hline Irlanda & 0,00 & 0,04 & - \\
\hline Israel & 0,18 & 0,20 & 0,09 \\
\hline Italia & - & 0,08 & 0,12 \\
\hline Japón & 0,12 & 0,12 & 0,15 \\
\hline Macau & - & - & 0,09 \\
\hline Nueva Zelanda & 0,00 & 0,16 & 0,03 \\
\hline Noruega & - & 0,16 & 0,12 \\
\hline Portugal & 0.06 & - & 0,06 \\
\hline Rusia & - & - & 0,03 \\
\hline Singapur & - & 0,04 & 0,15 \\
\hline Sudáfrica & 0,06 & - & - \\
\hline Suecia & 0,00 & 0,12 & 0,18 \\
\hline Suiza & 0,06 & 0,12 & 0,12 \\
\hline Taiwán & - & 0,08 & 0,03 \\
\hline Turquía & 0,12 & - & 0,00 \\
\hline Reino Unido & 0,29 & 0,32 & 0,42 \\
\hline
\end{tabular}

En la primera etapa, en la estructura de la red, se visualizan 4 nodos (Canadá, Estados Unidos, Francia y Reino Unido) y el resto de los países se visualizan como vértices (figura 3). En la segunda etapa, Estados Unidos y Reino Unido evolucionan y se convierten en hubs, mientras que Alemania, Bélgica, Israel, Holanda, Hong Kong, Nueva Zelanda y Noruega se convierten en nodos (figura 4). En la tercera etapa, Canadá y Francia evolucionan a hubs, Reino Unido gana reputación y crece el número de sus seguidores y se crea una subred adyacente. China, Corea, Dinamarca, España y Japón evolucionan a nodos. Finalmente, Israel retrocede de nodo a vértice (figura 5 ).

\section{Desarrollo de la red durante las etapas estudiadas}

En la tabla 4 se muestran los índices de densidad, conectividad, centralización, emisión y recepción para cada etapa. Como se puede observar, todos los índices muestran un incremento en sus valores, lo cual evidencia el avance sostenido de la conformación de la red de la comunidad científica de la disciplina en torno a la revista SMJ.

Tabla 4. Índices de la red para cada etapa.

\begin{tabular}{lcccccc}
\hline & $N^{\circ}$ Países & Densidad & Conectividad & Centralización & Recepción & Emisión \\
\hline Etapa I & 18 & 0,09 & 5,86 & 2,11 & 0,10 & 0,12 \\
\hline Etapa II & 26 & 0,12 & 9,70 & 3,61 & 0,10 & 0,14 \\
\hline Etapa III & 34 & 0,14 & 13,83 & 5,47 & 0,39 & 0,48 \\
\hline
\end{tabular}

En la tabla 5 se muestra la evolución de los índices de emisión y recepción para cada país durante las etapas en las que tienen participación. La posición sólida de Estados Unidos en el núcleo de la red durante las tres etapas se demuestra a través 
del crecimiento de su índice de emisión durante las 3 etapas. Como se ha venido enunciando anteriormente, este país ha jugado un papel importante en el desarrollo y el tránsito de otros países por las áreas de la estructura de la red, así como también en la incorporación de nuevos países a la misma.

Reino Unido, durante la etapa II evolucionó y se transformó en nodo y en la tercera se convierte en un hub diseminador de conocimientos, creando una sub-red en torno de sí en la estructura de la red en la zona semi-periferia. En esta posición contribuyó a la incorporación de autores de nuevos países, lo que la coloca en la etapa de expansión en la red. Esto queda evidenciado en la etapa III cuando su índice de emisión fue superior al índice de recepción $(0,15$ de índice de emisión y 0,14 de índice de recepción).

Tabla 5. Índices de emisión y recepción de cooperación de la red.

\begin{tabular}{|c|c|c|c|c|c|c|c|c|c|}
\hline \multirow[b]{2}{*}{ Países } & \multicolumn{3}{|c|}{ Autores por etapa } & \multicolumn{3}{|c|}{ Índice de emisión } & \multicolumn{3}{|c|}{ In ice de recepción } \\
\hline & $\begin{array}{c}\text { Etapa } \\
\text { I }\end{array}$ & $\begin{array}{c}\text { Etapa } \\
\text { II }\end{array}$ & $\begin{array}{c}\text { Etapa } \\
\text { III }\end{array}$ & $\begin{array}{c}\text { Etapa } \\
\text { I }\end{array}$ & $\begin{array}{c}\text { Etapa } \\
\text { II }\end{array}$ & $\begin{array}{l}\text { Etapa } \\
\text { III }\end{array}$ & $\begin{array}{c}\text { Etapa } \\
\text { I }\end{array}$ & $\begin{array}{l}\text { Etapa } \\
\text { II }\end{array}$ & $\begin{array}{c}\text { Etapa } \\
\text { III }\end{array}$ \\
\hline Alemania & 1 & 7 & 15 & 0 & 0 & 0,13 & 0 & 0,28 & 0,20 \\
\hline Argentina & 0 & 0 & 1 & 0 & 0 & 0 & 0 & 0 & 1 \\
\hline Australia & 0 & 3 & 13 & & 0 & 0 & & 0,33 & 0,07 \\
\hline Austria & 0 & 0 & 3 & & & 0 & & & 0 \\
\hline Bélgica & 3 & 5 & 7 & 0 & 0 & 0 & 0 & 0,4 & 0,42 \\
\hline Brasil & 0 & 0 & 2 & & & 0 & & & 0,50 \\
\hline Canadá & 36 & 35 & 66 & $0, .02$ & 0 & 0,21 & 0,13 & 0,4 & 0,36 \\
\hline Chile & 0 & 2 & 0 & 0 & 0 & & 0 & 1 & \\
\hline China & 0 & 1 & 10 & & 0 & 0,10 & & 1 & 0,40 \\
\hline Chipre & 0 & 0 & 3 & & & 0 & & & 0,33 \\
\hline Corea & 0 & 3 & 21 & & 0 & 0,09 & & 0.33 & 0,23 \\
\hline Dinamarca & 0 & 0 & 11 & & & 0,27 & & & 0,45 \\
\hline $\begin{array}{l}\text { Emiratos Árabes } \\
\text { Unidos }\end{array}$ & 0 & 0 & 4 & & & 0,25 & & & 0,25 \\
\hline Estados Unidos & 391 & 875 & 1.027 & 0,04 & 0,06 & 0,08 & 0 & 0 & 0 \\
\hline España & 3 & 4 & 34 & 0 & 0 & 0 & 0 & 0.33 & 0,28 \\
\hline Finlandia & 1 & 0 & 14 & 0 & & 0 & 0 & & 0,42 \\
\hline Francia & 19 & 43 & 51 & 0,05 & 0,06 & 0,19 & 0,47 & 0,44 & 0,45 \\
\hline Grecia & 0 & 1 & 3 & & 0 & 0 & & 1 & 0,33 \\
\hline Hong Kong & 0 & 6 & 37 & & 0 & 0,18 & & 0.50 & 0,10 \\
\hline Holanda & 6 & 17 & 11 & 0 & 0,11 & & 0 & 0,11 & \\
\hline India & 0 & 1 & 1 & & 0 & & & 0 & \\
\hline Indonesia & 0 & 1 & 0 & & 0 & & & 1 & \\
\hline Irlanda & 2 & 1 & 0 & 0 & 0 & & 0 & 1 & \\
\hline Israel & 6 & 11 & 5 & 0 & 0,09 & 0 & 0,16 & 0,27 & 0,10 \\
\hline Italia & 0 & 2 & 8 & & & 0 & & & 0,25 \\
\hline
\end{tabular}




\begin{tabular}{lccccccccc} 
Japón & 1 & 6 & 14 & 0 & 0 & 0 & 0,50 & 0,50 & 0,64 \\
\hline Macau & 0 & 0 & 2 & & & 0 & & 0 \\
\hline Nueva Zelandia & 1 & 5 & 3 & 0 & 0,20 & 0 & 0 & 0,20 & 0,33 \\
\hline Noruega & 0 & 8 & 6 & & 0 & 0 & 0,12 & 0,33 \\
\hline Portugal & 1 & 0 & 4 & 0 & & 0 & 1 & 0 \\
\hline Rusia & 0 & 0 & 1 & & & 0 & & 1 & 0,68 \\
\hline Singapur & 0 & 2 & 22 & & 0 & 0 & & 0 \\
\hline Sudáfrica & 1 & 0 & 0 & 0 & & & 1 & 0,10 & 0,18 \\
\hline Suecia & 1 & 10 & 16 & 0 & 0 & 0,06 & 0 & 0,55 \\
\hline Suiza & 2 & 4 & 9 & 0 & 0,25 & 0,11 & 1 & 0 \\
\hline Taiwán & 0 & 1 & 3 & & 0 & & 0 & 1 \\
\hline Turquía & 3 & 0 & 1 & 0 & 0 & & 0 & 0,14 \\
\hline Reino Unido & 47 & 68 & 70 & 0,08 & 0,08 & 0,15 & 0,10 & 0,10 & 0,14 \\
\hline
\end{tabular}

Canadá, Francia y Suecia mantuvieron un crecimiento estable de su índice de emisión, pero se mantuvo también el incremento del predominio en los valores del índice de recepción. Por esta razón Canadá y Francia se ubicaron en la posición de semi-periferia de la red y en la etapa de consolidación diseminación. Suecia se mantuvo en la periferia. El resto de los países muestra un índice de recepción muy superior al de emisión. Todos se ubican en la zona de la periferia en la estructura de la red y se mantienen en la etapa incorporación/ conformación.

Los patrones observados muestran que la tendencia será a que se continúe la transformación de vértices en nodos y de estos en hubs diseminadores de conocimientos que propicien la creación de sub-redes en torno suyo para la incorporación de nuevos países en la estructura de la red. Algunos de los países situados en la zona de periferia transitarán a la de semi-periferia, lo cual mostrará la evolución dinámica interna de la red de la comunidad científica mundial en torno a la generación y diseminación del conocimiento sobre la dirección estratégica.

\section{Evolución y transformación de la red}

En los apartados anteriores se planteó el proceso de incorporación, consolidación y transformación de los países en la estructura de la red en torno a la comunidad científica de la dirección estratégica. Para medir la transformación de la red de países en relación con cada etapa se empleó el índice de disimilitud de Coultier et. al. (1998) (ver tabla 6)

Tabla 6. Evolución de la red de la comunidad científica de la dirección estratégica.

\begin{tabular}{lll}
\hline & Etapa 2 & Etapa 3 \\
\hline Etapa 1 & 0,30 & 0,38 \\
\hline Etapa 2 & & 0,30 \\
\hline
\end{tabular}


Los resultados del cálculo de este índice muestra que la red de países en torno a la generación de conocimiento de la dirección estratégica de la primera a la segunda etapa tuvo un ascenso y un descenso de la segunda a la tercera. Este resultado se corresponde con el salto de la cantidad de autores incorporados en la segunda etapa propiciados por el incremento de la cantidad de números en cada volumen de la revista SMJ editados a partir del año 1988. En el caso de la tercera etapa la estratificación de la estructura de la red en 3 zonas y la creación de subredes adyacentes a los hubs influyen en este resultado.

\section{Significación de la cooperación de autores del país en el núcleo de la red en la incorporación y desarrollo de nuevos países}

Durante las etapas estudiadas, se muestra un crecimiento de la cantidad de autores de países que se incorporaron a la red y que dicha incorporación favoreció el incremento de la cantidad de autores procedentes de dichos países. Asimismo, se determinó que el crecimiento de los índices de emisión posibilitó la transformación de vértices a nodos y de nodos a hubs. También contribuyó a que países ubicados en la posición de periferia pasen a la semi-periferia como es el caso de Reino Unido Canadá y Francia. El próximo paso realizado será determinar la significación estadística de la evolución de estos países en la estructura de la red, el incremento del número de sus autores que publicaron artículos en SMJ y el nivel de cooperación del país del núcleo de la red con los mismos.

La cooperación, en el caso de la comunidad científica de la disciplina dirección estratégica, evidenció dos dimensiones: la primera dimensión es la cooperación entre autores de instituciones de un mismo país (intra-cooperación). En esta dimensión se publicaron 829 artículos en la SMJ durante el periodo de tiempo analizado. En la segunda dimensión se representa la cooperación entre autores de instituciones provenientes de países diferentes (inter-cooperación). En este caso, encontramos 245 artículos con autores de instituciones provenientes dos países, 20 artículos con la participación de autores de 3 países y 1 artículo con la participación de autores de 4 países. Como se puede observar en la tabla 7, en ambas dimensiones, la cooperación creció significativamente de una etapa a la siguiente $\mathrm{Si}$ bien la cantidad de artículos publicados en co-autoría entre instituciones dentro de un mismo país fue mayor.

Tabla 7. Distribución de frecuencias de la cooperación entre autores de uno o varios países

\begin{tabular}{lcccccccccc}
\hline & Artículo & Autores & $\begin{array}{c}\text { Aut } \\
\text { /Artic }\end{array}$ & Total & $\%$ & 1 País & 2 países & 3 países & 4 países & Total \\
\hline Etapa I & 326 & 525 & 1,61 & 166 & 50,92 & 144 & 21 & 1 & 0 & 166 \\
\hline Etapa II & 587 & 1,122 & 1,91 & 393 & 66,95 & 316 & 73 & 4 & 0 & 393 \\
\hline
\end{tabular}




\begin{tabular}{lcccccccccc}
\hline Etapa III & 703 & 1,498 & 2,13 & 536 & 76,24 & 369 & 151 & 15 & 1 & 536 \\
\hline Total & 1.616 & 3.145 & 1,95 & 1.095 & 67,76 & 829 & 245 & 20 & 1 & 1.095 \\
\hline
\end{tabular}

Los patrones de cooperación durante las 3 etapas revelan que el país situado en el núcleo posee un número mayor de artículos con la co-autoría de instituciones dentro del mismo país que la co-autoria con autores de otros países. Tendencia similar poseen los países que se ubican en la semi-periferia de la red. El crecimiento de los países que evolucionan hacia hubs dentro de la estructura de la red, sin embargo, es mayor.

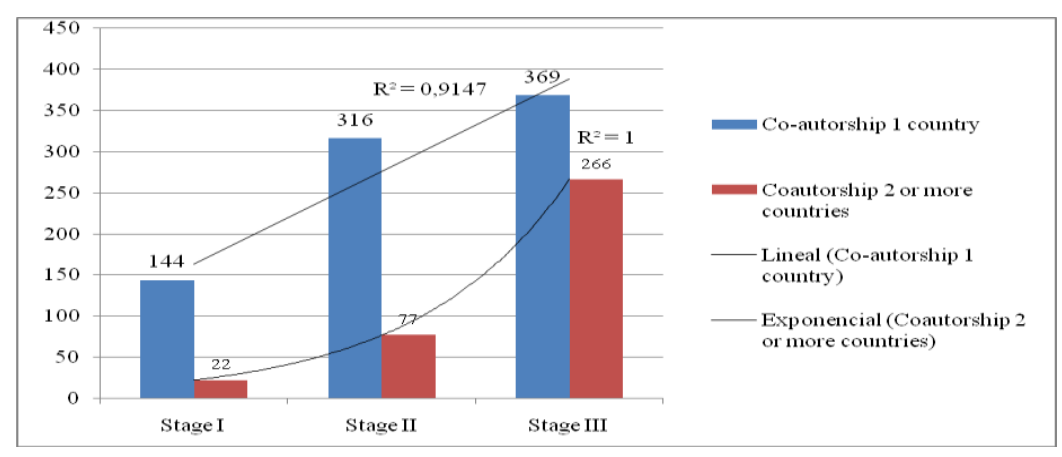

Figura 6. Tendencias en la co-autoría de artículos en la SMJ 1980-2009.

En la figura 6 se puede apreciar que a pesar de que el número de artículos publicados en cooperación entre autores de instituciones de un mismo país es mucho mayor que la cantidad de artículos publicados mediante la co-autoría de autores provenientes de instituciones de dos o más países, en este último caso el crecimiento es exponencial.

Pero mientras que el número de artículos co-autorados mediante intra-cooperación creció linealmente $\left(r^{2}=0,9147\right)$, el de los artículos en inter-cooperación creció de manera exponencial $\left(r^{2}=1\right)$. El número total de artículos crece de manera estable por etapas. Así, en la primera etapa encontramos 166, 393 en la segunda y 536 en la tercera. Este resultado es similar al reportado por Wagner y Leydesdorff (2005b) para la colaboración internacional y la formación de grupos.

La tabla 8 muestra la distribución de frecuencias de la cooperación entre autores dentro de un mismo país. Como se puede apreciar, existen 5 tendencias. La primera reúne a los países en los que el número de artículos publicados en intracooperación crece de manera estable durante las 3 etapas. En esta tendencia se sitúan Canadá, España, Estados Unidos, Italia, Japón, Reino Unido y Suecia. La segunda tendencia agrupa a los países en los que durante las dos primeras etapas la intra-cooperación se mantuvo estable y luego creció en la tercera etapa. Aquí encontramos a Alemania, Australia, Bélgica, Corea, Noruega y Turquía. La tercera tendencia recoge a los países que en la segunda etapa el nivel de intra-cooperación 
decrece y vuelve a subir en la tercera etapa. Aquí colocamos a Francia, Holanda, Israel y Nueva Zelanda.

Tabla 8. Distribución de frecuencias de la intra-cooperación de los países en la red

\begin{tabular}{|c|c|c|c|c|c|c|c|c|}
\hline \multirow{2}{*}{ Países } & \multicolumn{2}{|c|}{ Etapa I } & \multicolumn{2}{|c|}{ Etapa II } & \multicolumn{2}{|c|}{ Etapa III } & \multicolumn{2}{|c|}{ TOTAL } \\
\hline & Total & $\%$ & Total & $\%$ & Total & $\%$ & Total & $\%$ \\
\hline Alemania & 0 & $0,00 \%$ & 0 & $0,00 \%$ & 3 & $0,81 \%$ & 3 & $0,36 \%$ \\
\hline Argentina & 0 & $0,00 \%$ & 0 & $0,00 \%$ & 0 & $0,00 \%$ & 0 & $0,00 \%$ \\
\hline Australia & 0 & $0,00 \%$ & 0 & $0,00 \%$ & 3 & $0,81 \%$ & 3 & $0,36 \%$ \\
\hline Austria & 0 & $0,00 \%$ & 0 & $0,00 \%$ & 1 & $0,27 \%$ & 1 & $0,12 \%$ \\
\hline Bélgica & 1 & $0,69 \%$ & 1 & $0,32 \%$ & 1 & $0,27 \%$ & 3 & $0,36 \%$ \\
\hline Brasil & 0 & $0,00 \%$ & 0 & $0,00 \%$ & 0 & $0,00 \%$ & 0 & $0,00 \%$ \\
\hline Canadá & 9 & $6,25 \%$ & 4 & $1,27 \%$ & 8 & $2,17 \%$ & 21 & $2,53 \%$ \\
\hline Corea & 0 & $0,00 \%$ & 0 & $0,00 \%$ & 3 & $0,81 \%$ & 3 & $0,36 \%$ \\
\hline Chile & 0 & $0,00 \%$ & 0 & $0,00 \%$ & 0 & $0,00 \%$ & 0 & $0,00 \%$ \\
\hline China & 0 & $0,00 \%$ & 0 & $0,00 \%$ & 1 & $0,27 \%$ & 1 & $0,12 \%$ \\
\hline Chipre & 0 & $0,00 \%$ & 0 & $0,00 \%$ & 1 & $0,27 \%$ & 1 & $0,12 \%$ \\
\hline Dinamarca & 0 & $0,00 \%$ & 0 & $0,00 \%$ & 2 & $0,54 \%$ & 2 & $0,24 \%$ \\
\hline Emiratos Árabes & 0 & $0,00 \%$ & 0 & $0,00 \%$ & 1 & $0,27 \%$ & 1 & $0,12 \%$ \\
\hline Estados Unidos & 116 & $80,56 \%$ & 277 & $87,66 \%$ & 298 & $80,76 \%$ & 691 & $83,35 \%$ \\
\hline España & 1 & $0,69 \%$ & 1 & $0,32 \%$ & 10 & $2,71 \%$ & 12 & $1,45 \%$ \\
\hline Finlandia & 0 & $0,00 \%$ & 0 & $0,00 \%$ & 2 & $0,54 \%$ & 2 & $0,24 \%$ \\
\hline Francia & 4 & $2,78 \%$ & 7 & $2,22 \%$ & 4 & $1,08 \%$ & 15 & $1,81 \%$ \\
\hline Grecia & 0 & $0,00 \%$ & 0 & $0,00 \%$ & 0 & $0,00 \%$ & 0 & $0,00 \%$ \\
\hline Hong Kong & 0 & $0,00 \%$ & 0 & $0,00 \%$ & 2 & $0,54 \%$ & 2 & $0,24 \%$ \\
\hline Holanda & 2 & $1,39 \%$ & 3 & $0,95 \%$ & 1 & $0,27 \%$ & 6 & $0,72 \%$ \\
\hline India & 0 & $0,00 \%$ & 0 & $0,00 \%$ & 0 & $0,00 \%$ & 0 & $0,00 \%$ \\
\hline Indonesia & 0 & $0,00 \%$ & 0 & $0,00 \%$ & 0 & $0,00 \%$ & 0 & $0,00 \%$ \\
\hline Irlanda & 0 & $0,00 \%$ & 0 & $0,00 \%$ & 0 & $0,00 \%$ & 0 & $0,00 \%$ \\
\hline Israel & 1 & $0,69 \%$ & 2 & $0,63 \%$ & 1 & $0,27 \%$ & 4 & $0,48 \%$ \\
\hline Italia & 0 & $0,00 \%$ & 1 & $0,32 \%$ & 2 & $0,54 \%$ & 3 & $0,36 \%$ \\
\hline Japón & 0 & $0,00 \%$ & 1 & $0,32 \%$ & 2 & $0,54 \%$ & 3 & $0,36 \%$ \\
\hline Macau & 0 & $0,00 \%$ & 0 & $0,00 \%$ & 0 & $0,00 \%$ & 0 & $0,00 \%$ \\
\hline Nueva Zelanda & 0 & $0,00 \%$ & 1 & $0,32 \%$ & 0 & $0,00 \%$ & 1 & $0,12 \%$ \\
\hline Noruega & 0 & $0,00 \%$ & 2 & $0,63 \%$ & 2 & $0,54 \%$ & 4 & $0,48 \%$ \\
\hline Portugal & 0 & $0,00 \%$ & 0 & $0,00 \%$ & 2 & $0,54 \%$ & 2 & $0,24 \%$ \\
\hline Rusia & 0 & $0,00 \%$ & 0 & $0,00 \%$ & 0 & $0,00 \%$ & 0 & $0,00 \%$ \\
\hline Singapur & 0 & $0,00 \%$ & 0 & $0,00 \%$ & 1 & $0,27 \%$ & 1 & $0,12 \%$ \\
\hline Sudáfrica & 0 & $0,00 \%$ & 0 & $0,00 \%$ & 0 & $0,00 \%$ & 0 & $0,00 \%$ \\
\hline Suecia & 0 & $0,00 \%$ & 2 & $0,63 \%$ & 4 & $1,08 \%$ & 6 & $0,72 \%$ \\
\hline Suiza & 0 & $0,00 \%$ & 1 & $0,32 \%$ & 0 & $0,00 \%$ & 1 & $0,12 \%$ \\
\hline Taiwán & 0 & $0,00 \%$ & 0 & $0,00 \%$ & 0 & $0,00 \%$ & 0 & $0,00 \%$ \\
\hline Turquía & 1 & $0,69 \%$ & 0 & $0,00 \%$ & 0 & $0,00 \%$ & 1 & $0,12 \%$ \\
\hline Reino Unido & 9 & $6,25 \%$ & 13 & $4,11 \%$ & 14 & $3,79 \%$ & 36 & $4,34 \%$ \\
\hline Total & 144 & $100,0 \%$ & 316 & $100,0 \%$ & 369 & $100,0 \%$ & 829 & $100,0 \%$ \\
\hline
\end{tabular}

La cuarta tendencia es la que recoge a los países en los que su intra-cooperación se mantuvo superior en las dos primeras etapas y decrece en la tercera. Aquí incluimos a Austria, China, Chipre, Dinamarca, Finlandia, Hong Kong, Portugal, 
Singapur y Emiratos Árabes Unidos. Finalmente, en la quinta tendencia se agrupan los países que no muestran ningún nivel del intra-cooperación durante las tres etapas: Argentina, Brasil, Chile, Grecia, Indonesia, Irlanda, Macau, Rusia, Sudáfrica y Taiwán.

Para medir la significación de la cooperación de autores del país ubicado en el núcleo de la estructura de la red en relación con la inclusión de autores de los países que mostraron un crecimiento estable durante las tres etapas, se comparó la cantidad de artículos publicados de manera cooperada con la participación de autores del país ubicado en el núcleo con la cantidad de autores participantes en la cooperación inter-países ubicados en otras posiciones de la red.

Para la selección de los países para determinar la significación de la intercooperación se tuvieron en cuenta dos criterios: 1) que el país elegido debió pertenecer a la red de la comunidad científica de la disciplina dirección estratégica durante las 3 etapas analizadas y 2) que el país haya tenido un crecimiento en el número de publicaciones en la SMJ durante las etapas analizadas.

Para la observación se escogieron 3 países que mostraron crecimiento en el número de investigadores que publicaron artículos en SMJ de manera cooperada con autores procedentes del país que se ubica en el núcleo de la red. Los países escogidos fueron Canadá, Francia y Reino Unido (tabla 9A). Además se escogieron 3 países que mostraron crecimiento durante las tres etapas y que mostraron niveles discretos de participación de sus autores en cooperación con autores del país ubicado en el núcleo de la red. Los países escogidos fueron Alemania, Bélgica y España (tabla 10A). El nivel de significación estadística se determinó mediante el cálculo de la prueba $t$ pareada, mediante el software SigmaStat $\vee 3.5$ para Windows.

Tabla 9 A. Grupo de países con un incremento de las publicaciones en la SMJ e inter-cooperación con autores del país en el núcleo de al red.

\begin{tabular}{lccccccc}
\hline \multirow{2}{*}{ Países } & \multicolumn{2}{c}{ Etapa I } & \multicolumn{2}{c}{ Etapa II } & \multicolumn{2}{c}{ Etapa III } \\
\cline { 2 - 8 } & Cooperación & Artículos & Cooperación & Artículos & Cooperación & Artículos \\
\hline Canadá & 2 & 36 & 13 & 35 & 21 & 66 \\
\hline Francia & 9 & 19 & 16 & 43 & 20 & 51 \\
\hline Reino Unido & 5 & 47 & 7 & 68 & 10 & 70 \\
\hline
\end{tabular}

Tabla 9 B. Significación estadística artículos publicados SMJ y inter-cooperación con el país en el núcleo de la red.

\begin{tabular}{lccccc}
\hline Treatment Name & N & Missing & Mean & Std Dev & SEM \\
\hline Col 2 & 3 & 0 & 62,333 & 10,017 & 5,783 \\
\hline Col 1 & 3 & 0 & 34 & 14,107 & 8,145 \\
\hline Difference & 3 & 0 & 28,333 & 4,726 & 2,728 \\
\hline
\end{tabular}


$t=10.384$ con 2 grados de libertad. $(p=0.009) .95 \%$ de intervalo de confianza para la diferencia de las medias: 16.594 a 40.073.

El resultado muestra que el incremento de la cantidad de artículos publicados en la SMJ con la co-autoría de autores de instituciones del país ubicado en el núcleo de la red posee un nivel de significación estadística altamente significativo $(p=0,009)$, lo cual implica que cuanto mayor es la cooperación con autores provenientes del país ubicado en el núcleo de la red, mayor es también la posibilidad de publicación de artículos en la revista SMJ. En la medida en que se incrementó el índice de emisión del país ubicado en el núcleo de la red con los países observados, estos se desarrollaron y mejoraron su posición en la estructura de la red de una etapa a la siguiente.

Tabla 10 A. Grupo de países con un incremento de las publicaciones en la SMJ y poca inter-cooperación con autores del país en el núcleo de la red.

\begin{tabular}{lccccccc}
\hline \multirow{2}{*}{ Países } & \multicolumn{2}{c}{ Etapa I } & \multicolumn{2}{c}{ Etapa II } & \multicolumn{2}{c}{ Etapa III } \\
\cline { 2 - 8 } & Cooperación & Artículos & Cooperación & Artículos & Cooperación & Artículos \\
\hline Alemania & 0 & 1 & 1 & 7 & 2 & 15 \\
\hline Bélgica & 0 & 3 & 1 & 5 & 1 & 7 \\
\hline España & 0 & 3 & 1 & 4 & 9 & 34 \\
\hline
\end{tabular}

Tabla 10 B. Significación estadística artículos publicados SMJ y baja intercooperación con el país en el núcleo de la red.

\begin{tabular}{|c|c|c|c|c|c|c|}
\hline Treatment Name & $\mathrm{N}$ & & Missing & Mean & Std Dev & SEM \\
\hline Col 1 & & 3 & 0 & 2,333 & 1,155 & 0,667 \\
\hline Col 2 & & 3 & 0 & 18,667 & 13,868 & 8,007 \\
\hline Difference & & 3 & 0 & $-16,33$ & 13,65 & 7,881 \\
\hline
\end{tabular}

$t=-2.072$ con 2 grados de libertad. $(p=0.174)$. Prueba realizada para con un alpha $=$ 0.050: 0.193. $95 \%$ de confianza intervalo de diferencia de las medias: -50.243 a 17.576

Los resultados obtenidos no muestran significación estadística al lograrse un valor de $p=0,174$ para un intervalo de confianza alpha $=0,050: 0,193$. El valor de la prueba realizada $(0,174)$ es menor que el valor esperado 0,800 . Por tanto, para estos tres países que crecieron en el número de autores que participaron en la red y que no se aprecia crecimiento en los índices de cooperación se observa que se mantuvieron en la posición de periferia de la red, mantuvieron su estadio en la etapa de conformación de la red y no evolucionaron significativamente (Tablas 10A y 10B). El resultado confirma que la cooperación de autores de instituciones de países ubicados en las áreas semi-periferia y periferia con autores del núcleo de la red favorece su desarrollo y transformación dentro de la estructura de la red.

Otro elemento que evidencia la importancia de la colaboración en el desarrollo de la comunidad científica de la dirección estratégica en torno a la SMJ es la inserción de nuevos participantes en la estructura de la red. En la etapa I el $33.33 \%$ de los 
países que participaron en la red fueron introducidos mediante la co-autoría con autores procedentes de instituciones de los países con mayor desarrollo, principalmente el ubicado en el núcleo y los países con mayor participación ubicados en la periferia. Estos países sirvieron de puente para los países entrantes.

En la etapa II el porcentaje de países incluidos en la estructura de la red a través de la cooperación fue el $83.33 \%$. En el $100 \%$ de estas incorporaciones participaron autores del país del núcleo de la red. En dos casos participaron, además, autores de instituciones de Francia y del Reino Unido.

En la etapa III, el $87.5 \%$ de los países incorporados fue a través de la cooperación. El $42.85 \%$ de los países incorporados fue a través de la cooperación con el país ubicado en el núcleo, el $42.85 \%$ con países ubicados en la semi-periferia (Reino Unido y Canadá) y el $14.28 \%$ mediante la cooperación con un país en la periferia. En esta etapa también se reincorporan a la estructura de la red 3 países que estuvieron ausentes en la segunda etapa, dos de estos reaparecieron mediante la cooperación.

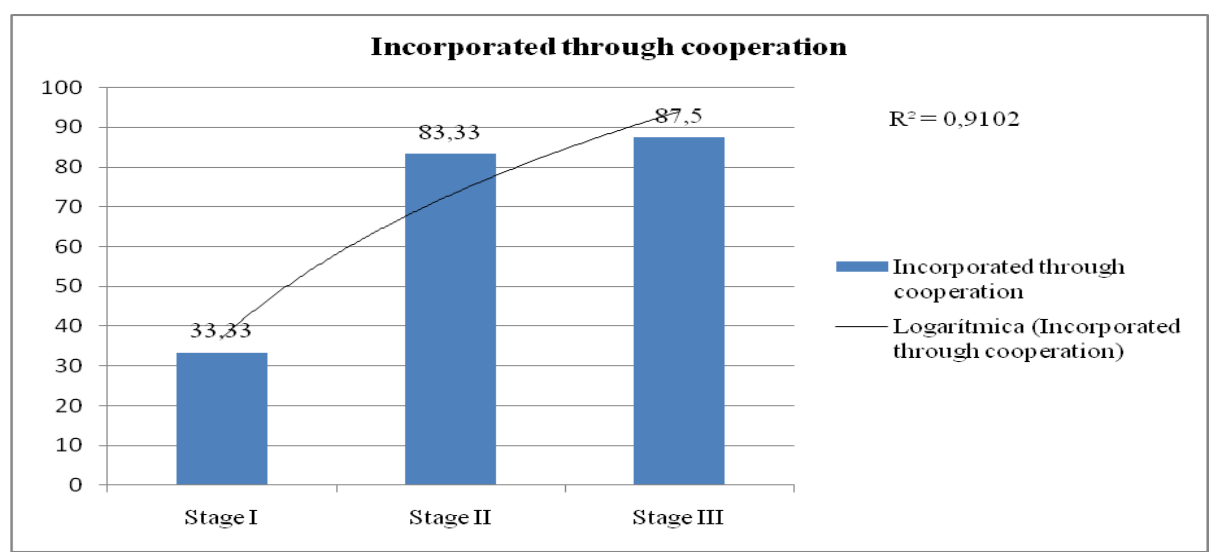

Figura 7. Porcentaje de países que fueron incorporados a través de la cooperación.

Como se puede apreciar en la figura 7 existió un incremento sostenido de la cantidad de países que se incorporaron a la estructura de la red a través de la cooperación, principalmente con el país ubicado en el núcleo. En la tercera etapa se incrementó el porcentaje de países incorporados a través de la cooperación de los países en la semi-periferia. Este resultado evidencia que los países que consolidan su posición en esta área de la red comienzan a ganar seguidores, lo que contribuye a consolidar este estrato. 


\section{Conclusiones}

Los resultados obtenidos en el estudio evidencian que la estructura de la red de la comunidad científica en dirección estratégica en torno a la revista SMJ transitó por 3 etapas: (1) conformación/incorporación; (2) consolidación/diseminación y (3) expansión/transformación. Las etapas se determinan a partir del patrón de características que se observan en la estructura de la red sobre la base de la evolución de las variables densidad, conectividad, clusterabilidad, índice de recepción y de emisión. Además, se tiene en cuenta la posición de los países en la estructura de la red, el patrón de las relaciones entre los países ubicados en el núcleo de la red con los países ubicados en la semi-periferia o la periferia y la relación entre los países ubicados en la misma área: periferia-periferia o semiperiferia - semi-periferia.

Las etapas descritas pueden ser válidas para estudiar la evolución de redes académicas de otras disciplinas o subcampos dentro de la dirección de empresas. Cabe suponer que el comportamiento de la evolución de los actores por los diferentes estadios será cíclico. Esto es, se incorporan nuevos países que transitarán por las etapas de evolución. Además, es previsible que se incorporen nuevos países en el núcleo de la estructura de la red y así sucesivamente.

La creación de los índices de emisión y de recepción posibilita medir el desarrollo de un país dentro de la estructura de la red y determinar el posible momento en que se convertirá en hub durante su tránsito por algunas de las etapas de evolución de una red académica.

En la tercera etapa, según se consolidan los países en el área de semi-periferia comienzan a ganar seguidores, lo que trae consigo la transformación de dichos países en hubs y se crean subredes diseminadoras de conocimiento en torno a los mismos. Esto influye significativamente en el crecimiento de la red y en la diseminación de conocimientos sobre dirección estratégica por el mundo, así como coadyuva a ubicar a la comunidad científica de la dirección estratégica en la fase de expansión/ transformación.

Los países cuyos autores contaron con la cooperación de autores procedentes del país ubicado en el núcleo de la red, mostraron mayor nivel de desarrollo que países que, aunque crecieron, no contaron con dicha cooperación. Los resultados muestran que en la medida que crece la cooperación en la publicación de artículos en la revista SMJ crece también la cantidad de autores de otros países incorporados a la red de la comunidad científica de la dirección estratégica y aumenta el poder de influencia de la revista en el desarrollo de la disciplina. 
Países ubicados en la zona de semi-periferia de la red que mantuvieron un incremento de la cantidad de artículos publicados co-autorados con autores del país en la zona núcleo de la red, en la tercera etapa, se convirtieron en hubs y ganaron seguidores y contribuyeron a la incorporación de nuevos países a la red.

Todos los índices empleados en el estudio para evaluar la dinámica de la evolución del desarrollo de la red de la comunidad científica de la dirección estratégica a través de las publicaciones en la SMJ mostraron un incremento estable y sostenido durante las 3 etapas, lo cual evidencia la sostenibilidad de la red de esta comunidad científica y el incremento progresivo de su influencia en el campo de la dirección de empresas en general.

Existe una mayor fuerza de cooperación entre los autores de países ubicados en el núcleo de la red con los países ubicados en la zona semi-periferia que los autores de países ubicados en la semi-periferia con los autores de países de la periferia de la red.

Los resultados obtenidos en la presente investigación podrían complementarse con otros estudios relacionados con la estructura de cooperación de las redes de investigación inter-universidades. También sería interesante estudiar el papel de las universidades y escuelas de negocios en el desarrollo de la disciplina en Estados Unidos, ya que es el país determinante al ser el único en el núcleo de la red. También sería interesante observar la evolución futura de la transformación de la estructura de la red pasados otros años de publicación de la revista SMJ. Finalmente, la metodología seguida permitiría la realización de estudios similares con otras revistas u otros campos de la dirección de empresas y su comparación con el presente resultado.

\section{Bibliografía}

Andrews, K. (1971). The Concept of Corporate Strategy. Homewood, Illinois: DowJones-Irwin.

Ansoff, I.H. (1965). Corporate Strategy: An Analytic Approach to Business Policy for Growth and Expansion. New York: McGraw Hill.

Azar, O.H Brock, D.M. (2008). A citation ranking of strategic management journals. Journal of Economics \& Management Strategy, 17(3781-802).

Batagelj, V., \& Mrvar, A. (1996). Pajek (Version 1.26) [Free for noncommercial use]: Vladimir Batagelj and Andrej Mrvar.

Burt, R. (2001). Attachment, Decay, and Social Network. Journal of Organizational Behavior, 22(6), 619-643.

Burt, R. (2007). Secondhand Brokerage: Evidence on the Importance of Local Structure for Managers, Bankers, and Analysts. Academy of Management Journal, $\mathbf{5 0}(1), 119-148$. 
Burt, R. (2008). Information and Structural Holes: Comment on Reagan's and Zuckerman. Industrial and Corporate Change, 17(5), 953-969.

Callon, M., Courtial, J.P., \& Laville, F. (1991). Co-Word Analysis as a Tool for Describing the Network of Interactions Between Basic and Technological Research: The Case of Polymer Chemestry. Scientometrics, 22(1), 155-205.

Chandler, A. (1962). Strategy and Structure: Chapters in the History of American Industrial Enterprise. Cambridge, Massachusetts: M.I.T Press.

Coultier, N., Monarch, I., \& Konda, S. (1998). Software Engineering as Seen Through its Research Literature: A Study in Co-Word Analysis. Journal of the American Society for Information Science, 49(13), 1206-1223.

De Nooy, W., Mrvar, A., Batagelj, V. (2005) Exploratory Social Network Analysis with Pajek. New York, Cambridge University Press.

Eldredge, D., \& Galloway, R. (1983). Study of the Undergraduate Business Policy Course at AACS B- Accredited Universities. Strategic Management Journal, 4(1), 85-90

Feurer, R., \& Chaharbaghi, K. (1995). Strategy development: Past, present and future. Management Decision, 33(6), p: 11.

Franke, R.H., Edlund, T.W., \& Oster, F. (1990). The development of strategic management: Journal quality and article impact. Strategic Management Journal, 11(3), 243-253.

Fréry, F. (2006). The Fundamental Dimensions of Strategy. MIT Sloan Management Review, 48(1), p.71.

Furrer, O., Thomas, H., \& Goussevskaia, A. (2008). The Structure and Evolution of the Strategic Management Field: A Content Analysis of 26 Years of Strategic Management Research. International Journal of Management Reviews, 10(1), 1-23.

Gordon, R.A., \& Howell, J.E. (1959). Higher Education for Business. New York: Columbia University Press.

Gulati, R. (1998). Alliances and Networks. Strategic Management Journal, 19(4), 293-317.

Gulati, R., Nitin, N., Zaheer, A. (2000). Strategic Networks. Strategic Management Journal, 21(3), 203-215.

Hahn, E., \& Doh, J. (2006). Using Bayesian Methods in Strategy Research: An Extension of Hansen et al. Strategic Management Journal, 27, pp 783-798.

Hoetker, G. (2006). The Use of Logit and Probit Models in Strategic Management Research: Critical Issues. Strategic Management Journal, 28(2), 331-343.

Hofer, C., \& Schendel, D. (1978). Strategy Formulation: Analytical Concepts: West Publishing Company.

Hulland, J. (1999). Use of partial least squares (PLS) in strategic management research: A Review of Four Recent Studies. Strategic Management Journal, 20(2), p: 195.

Kamada, T., \& Kawai, S. (1989). An algorithm for drawing general undirected graphs. Information Processing Letters, 31(1), 7-15.

Katz, J.S. (1994) Geographical proximity and scientific collaboration. Scientometrics, 31(1) 31-43.

Ketchen, D., \& Shook, C. (1996). The Application of Cluster Analysis in Strategic Management Research: An Analysis and Critique. Strategic Management Journal, 17(6), P: 441. 
Kogut, B. (2000). The Network as Knowledge: Generative Rules and the Emergence of Structure. Strategic Management Journal, 21(3), 405-425.

Learned, E., Christensen, R., Andrews, K., \& Guth, W. (1965). Business Policy: Text and Cases. Homewood, Illinois: Richard D. Irwin, Inc.

Nag, R., Hambrick, D.C. \& Chen, M.H. (2007). What is Strategic Management, Really? Inductive Derivation of a Consensus Definition on the Field. Strategic Management Journal, 28(3): 935 - 955.

Nerur, S.P., Rasheed, A.A. \& Natarajan, V. (2008). The Intellectual Structure of the Strategic Management Field: An Author Co-Citation Analysis. Strategic Management Journal, 29(11), 319-336.

Peters H.P.F., Van Raan A.F.J. (1993a). Co-word-based Maps of Chemical Engineering. Part I: Representations by Direct Multidimensional Scaling, Research Policy, 22(1): 23-46.

Peters H.P.F., Van Raan A.F.J. (1993b). Co-word-based Maps of Chemical Engineering. Part II: Representations by Combined Clustering and Multidimensional Scaling, Research Policy, 22(1): 47-72.

Phelan, S.E., Ferreira, M., \& Salvador, R. (2002). The First Twenty Years of Strategic Management Journal. Strategic Management Journal, 23(12), 11611168.

Podsakoff, P.M., MacKenzie, S.B., Bachrach, D.G., \& Podsakoff, N.P. (2005). The Influence of Management Journals in the 1980s and 1990s. Strategic Management Journal, 26(5), 473-488.

Porter, M.E. (1980). Competitive Strategy. Free Press: NewYork.

Porter, M.E. (1985). Competitive Advantage. Free Press: New York.

Ramos-Rodriguez, A.R., Ruiz-Navarro, J. (2004). Changes in the Intellectual Structure of Strategic Management Research: A Bibliometric Study of the Strategic Management Journal, 1980-2000. Strategic Management Journal, 25(10), 9811004.

Summer, C., Bettis, R.A., Duhaime, I.H., Grant, J.H., Hambrick, D.C., Snow, C.C., et al. (1990). Doctoral Education in the Field of Business Policy and Strategy. Journal of Management, 16(2), 361-398.

Tahai, A., Meyer, M.J. (1999). A Revealed Preference Study of Management Journals' Direct Influences. Strategic Management Journal, 20(3), 279-296.

Wagner, C.S., Leydesdorff, L. (2005a). Mapping the network of global science: comparing international co-authorships from 1990 to 2000. International Journal of Technology and Globalisation, 1(2), 185-208.

Wagner, C.S., Leydesdorff, L. (2005b). Network Structure, Self Organization and the Growth of International Collaboration in Science. Research Policy, 34(10), 1608-1618.

Zajac, E.J. (1998). Commentary on "Alliances and Networks" By R. Gulati. Strategic Management Journal, 19(4), 319-321.

\section{Agradecimientos}

Los autores desean agradecer al profesor Gabriel Velez, editor del monográfico, al profesor Isidro Maya-Jariego, al profesor Emilio López-Zapata y a dos árbitros anónimos por sus sugerencias constructivas y sus intuitivos comentarios. Este trabajo ha sido financiado por el proyecto ECO2009-10358 del Ministerio de Ciencia 
e Innovación (España) y la Cátedra Iberdrola de Investigación en Dirección y Organización de Empresas. 